Reprint 776

Applications of an Infrared Doppler Lidar in Detection of Wind Shear

C. M. Shun \& P. W. Chan

Journal of Atmospheric and Oceanic Technology, volume 25, issue 5, p.637 - 655 


\title{
Applications of an Infrared Doppler Lidar in Detection of Wind Shear
}

\author{
C. M. SHun AND P. W. Chan \\ Hong Kong Observatory, Kowloon, Hong Kong, China
}

(Manuscript received 27 August 2007, in final form 19 November 2007)

\begin{abstract}
In December 2005, operational wind shear alerting at the Hong Kong International Airport (HKIA) reached an important milestone with the launch of the automatic Lidar (light detection and ranging) Windshear Alerting System (LIWAS). This signifies that the anemometer-based and radar-based wind shear detection technologies deployed worldwide in the twentieth century have been further advanced by the addition of the lidar-a step closer to all-weather coverage.

Unlike the microburst and gust front, which have a well-defined coherent vertical structure in the lowest several hundred meters of the atmosphere, terrain-induced wind shear tends to have high spatial and temporal variability. To detect the highly changeable winds to be encountered by the aircraft under terraininduced wind shear situations, the Hong Kong Observatory devises an innovative glide path scan (GPScan) strategy for the lidar, pointing the laser beam toward the approach and departure glide paths, with the changes in azimuth and elevation angles concerted. The purpose of the GPScans is to derive the headwind profiles and hence the wind shear along the glide paths. Developed based on these GPScans, LIWAS is able to capture about $76 \%$ of the wind shear events reported by pilots over the most-used approach corridor under clear-air conditions. During the past two years, further developments of the lidar took place at HKIA, including the use of runway-specific lidar to further enhance the wind shear detection performance.
\end{abstract}

\section{Introduction}

Since the opening of Hong Kong International Airport (HKIA) in August 1998, about 1 in 500 flights at the airport encountered significant wind shear. In early 2007 , the number of aircraft movements, that is, approaches and departures, at HKIA reached 800 per day, implying that on average between one to two pilot reports of wind shear are received each day. Provision of timely and accurate wind shear alerts to the aircraft is thus a priority development area of the aviation weather services for HKIA.

HKIA is situated in a coastal environment with complex orography. Apart from the thunderstorm-induced microburst and gust front, which are covered by the terminal Doppler weather radar (TDWR) (Biron et al. 1990; Michelson et al. 1990; Shun and Johnson 1995), and wind shear brought by the sea breeze (Lee and Shun 2003), which are covered by a network of anemometers and weather buoys, low-level terrain-

Corresponding author address: P. W. Chan, 134A Nathan Road, Kowloon, Hong Kong, China.

E-mail: pwchan@hko.gov.hk

DOI: 10.1175/2007JTECHA1057.1 induced wind shear in nonrainy weather conditions accounts for most of the pilot reports at HKIA. Recognizing the need to supplement the TDWR and anemometer network in capturing these clear-air events, the Hong Kong Observatory (HKO) introduced in mid-2002 an infrared coherent Doppler lidar (light detection and ranging) operating at $2-\mu \mathrm{m}$ wavelength to HKIA, on an experimental basis, for wind shear alerting (Shun and Lau 2002). It was the first operational lidar for round-the-clock airport weather alerting in the world. Before 2002, Doppler lidars had only been used at airports for collecting data in wake turbulence studies (Thomson and Hannon 1995; Hannon et al. 1994) or in field experiments of mesoscale meteorology (Banta et al. 1993), mountain meteorology (Banta et al. 1990; Bougeault et al. 2001), and demonstrations of wind shear detection on board aircraft (Woodfield and Vaughan 1983; Hannon et al. 1999). Indeed, Mahapatra (1999) only mentioned "possible use for visibility ceiling (cloud base height) and fog and visibility quantification" of the lidar in aviation weather surveillance applications.

In aviation meteorology, wind shear refers to a sustained change (i.e., lasting more than a few seconds as 


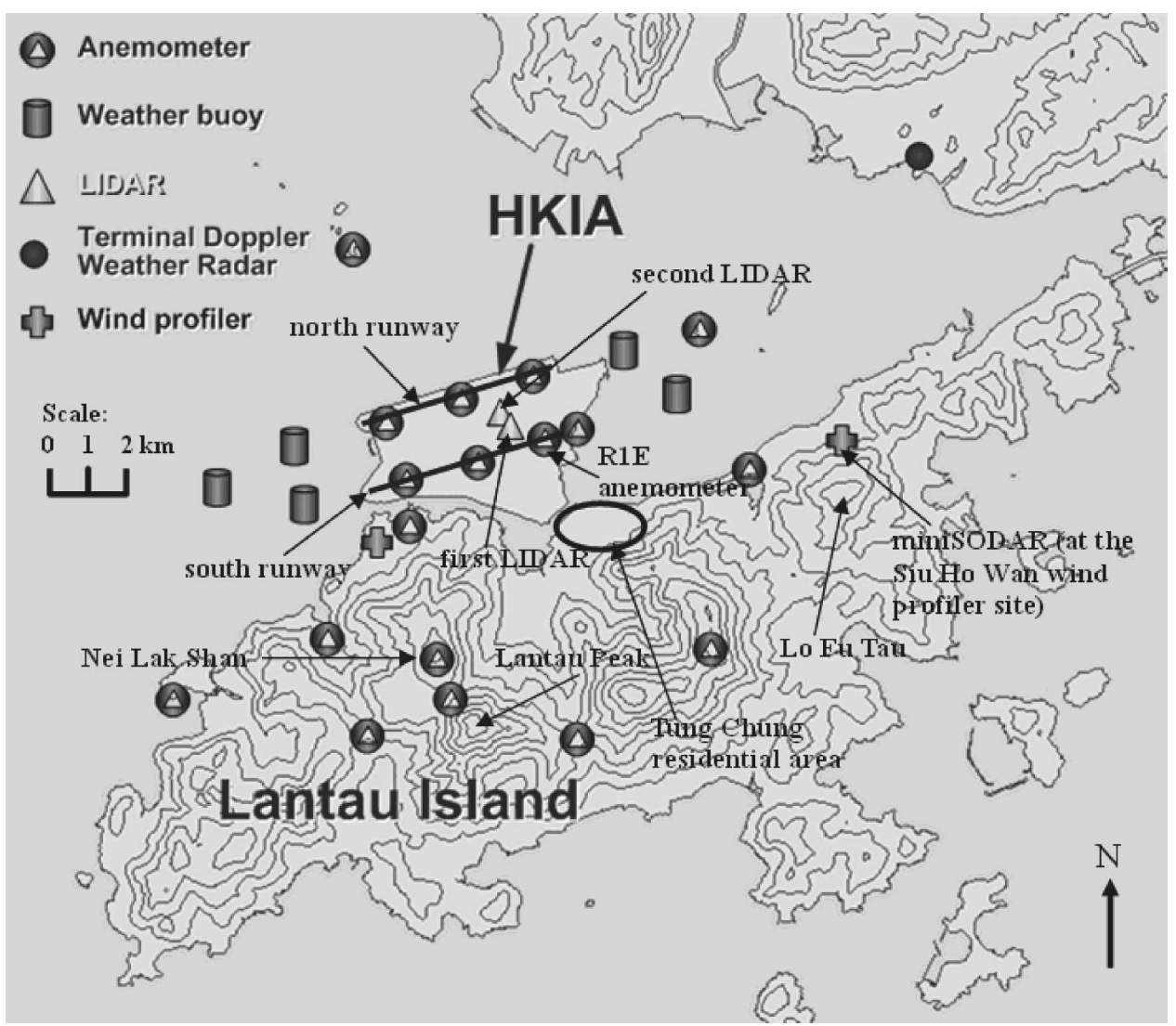

FIG. 1. Geographical environment of HKIA and meteorological instruments for wind shear alerting. Height contours are in $100 \mathrm{~m}$.

experienced by the aircraft) in the headwind/tailwind, resulting in a change in the aircraft lift. A change of 15 $\mathrm{kt}$ or more in the headwind/tailwind is considered to be significant wind shear, which may require timely and corrective action by the pilot so that the aircraft could maintain its intended flight path.

Wind shear could occur in a wide spectrum of weather conditions, from a stably stratified boundary layer in the presence of terrain effects, to sea breeze in generally fine weather, to microburst and gust front associated with severe convection (ICAO 2005). The complex orography around HKIA poses a unique challenge to the detection and alerting of low-level wind shear, that is, wind shear below $1600 \mathrm{ft}$ and within $3 \mathrm{n}$ $\mathrm{mi}$ from the runway thresholds (ICAO 2007). Figure 1 shows the geographical situation of HKIA. The airport was built on Chek Lap Kok Island, which was reclaimed from the sea. The most prominent topography around HKIA is the mountainous Lantau Island to its south, having peaks rising to nearly $1000 \mathrm{~m}$ above ground level (AGL) and valleys as low as $400 \mathrm{~m}$ AGL. The airport is surrounded by sea on the other three sides. To the northeast of HKIA, there are also a number of smaller hills with peaks of about $600 \mathrm{~m}$ AGL.

Located on the coast of southern China, the prevailing wind in Hong Kong comes from the east. This is particularly so in springtime when southern China is under the influence of the northeast monsoon. The atmosphere in springtime is usually stably stratified, frequently with low-level temperature inversions, with the wind veering from easterly at the surface to southerly on the hilltop level ( $\sim 1000 \mathrm{~m}$ AGL). Under such conditions, airflow disturbances including gravity waves and vortices could be generated over the airport area. During outbreaks of cold air from the north in winter and early spring, the strong northeasterly airstream can also be disrupted by the hills to the northeast of HKIA. In summer, airflow disruption by Lantau Island occurs in the southwest monsoon and during passages of tropical cyclones. As a result, terrain-induced wind shear is the major type of low-level wind shear at HKIA, accounting for about $70 \%$ of all the pilot wind shear reports. Sea breeze stands as the second most frequent cause of wind shear, bringing about $20 \%$ of the cases. 
The remaining events mostly occur in severe convective weather even though the percentage, based on pilot reports, might have been underestimated due to the general practice of pilots to avoid severe convection during approach and take-off, especially when an alert of the microburst was issued by TDWR. Low-level jets in the northeast monsoon could also bring about significant wind shear to the departing aircraft, but they are rather infrequent.

\section{Limitations of conventional wind shear detection systems}

Conventional wind shear detection technologies involve the use of the ground-based anemometer network, weather radar, and wind profiler. Following several aircraft accidents during the mid-1970s, the anemometer-based Low-Level Windshear Alerting System (LLWAS) was developed and installed at over 100 aerodromes in the United States (ICAO 2005). Similar anemometer-based systems were deployed in a number of international airports, for example, in late 1970s at the old HKIA at Kai Tak in Hong Kong, and recently at the new Suvarnabhumi Airport in Bangkok, Thailand. LLWAS was originally intended to detect the gust front. At HKIA, this anemometer-based technology was extended by deploying weather buoys over the sea surrounding the airport island for early detection of wind shear brought about by the sea breeze. The performance of the anemometer-based systems is considered reasonable for shear associated with low-level convergence lines having vertical extent in the lowest couple hundred meters.

In the United States, LLWAS was extended to cover the microburst, which was found to be the major wind shear hazard caused by thunderstorms in the 1980s. In this respect, LLWAS is considered limited because it can only detect the horizontal wind shear at ground level, even with the various enhancements to increase the density and coverage of the anemometers, as well as the wind shear detection algorithms for identification of the divergence wind patterns. This limitation is particularly the case for terrain-induced wind shear with high spatial and temporal variability. Gusty wind conditions, less-than-ideal anemometer siting, and data quality issues are also known to bring false alarms to LLWAS.

Developed specifically to detect the microburst and gust front in the 1980s and early 1990s, the C-band TDWR with narrow beam $\left(\sim 0.5^{\circ}\right.$ half-power beamwidth) was deployed by the U.S. Federal Aviation Administration (FAA) at 45 U.S. airports. Similar C-band weather radar systems were also deployed at international airports in Japan, Singapore, Malaysia, and Ko- rea starting in the mid-1990s. At HKIA, a TDWR, with some local enhancements to the FAA system, was installed at about $12 \mathrm{~km}$ to the east-northeast of the airport (Fig. 1) in 1996 (Shun and Johnson 1995). It has proved to be effective in the warning of low-level wind shear in rain, for example, during the passage of thunderstorms and tropical cyclones. At HKIA, however, the majority of terrain-induced wind shear occurs in springtime under nonrainy weather. Unfortunately, clear-air returns are not always available from TDWR. Even when they are available, the Doppler velocity data are not of consistently good quality for wind shear recognition by automatic algorithms. Similar situations are encountered by TDWRs installed at airports over the western mountains of the United States (Keohan 2007).

Keohan (2007) also reported warnings of terraininduced wind shear and turbulence using wind profilers and an anemometer network at the Juneau airport in Alaska. Regression equations derived from aircraft measurements of turbulence in relation to the wind profiler and anemometer sensor information are used to generate warnings of moderate and severe turbulence. A similar system is being operated at HKIA to alert terrain-induced turbulence, but data quality issues with the wind profiler data and representativeness of the wind profiler and anemometer data relative to the actual wind conditions along the glide paths continue to be a limiting factor in improving the performance of the system.

\section{Doppler lidar at HKIA}

To address the above difficulties with the conventional technologies in the detection of terrain-induced wind shear in dry weather, HKO introduced an infrared coherent Doppler lidar to HKIA (Shun and Lau 2002) in August 2002, the first of its kind for aviation weather alerting in the world. The lidar was installed on the rooftop of the air traffic control complex near the center of HKIA (labeled as the "first lidar" in Fig. 1), commanding a good view of the approach/departure corridors of the two parallel runways. It operates at a wavelength of $2 \mu \mathrm{m}$ with pulse energy of about $2 \mathrm{~mJ}$. The pulse repetition frequency is $500 \mathrm{~Hz}$, and line-ofsight data are output at $10 \mathrm{~Hz}$ (i.e., an average of 50 pulses for each datum). With a range resolution of about $100 \mathrm{~m}$ and 1024 range bins, the measurement distance starts from about $400 \mathrm{~m}$ and is up to $10 \mathrm{~km}$. The maximum unambiguous velocity is normally 20 $\mathrm{m} \mathrm{s}^{-1}$, extendable to $40 \mathrm{~m} \mathrm{~s}^{-1}$ at the expense of the range. A second lidar of the same model was installed in 2006 (see section 10). In the following discussions, 
the "lidar" refers to the "first lidar" in Fig. 1 unless otherwise specified.

The lidar technology is well suited for detecting terrain-induced wind shear at HKIA as it possesses the following characteristics:

(i) Measurement capability-the presence of abundant aerosols in the airport area and the drying up of the air by the föhn effect after climbing over the mountains provide a suitable environmental condition for wind measurements by the lidar.

(i) Scanning flexibility-compared to a conventional weather radar, the lidar has a smaller scanner (which is functionally equivalent to the antenna of the weather radar), which allows more flexibility in designing the scan strategy. This enables more precise depiction of the complicated, threedimensional flow structure in terrain-induced disturbances, particularly along the glide paths [see discussion on the glide path scans (GPScans) in section 7].

(iii) High spatial resolution-with a range resolution of about $100 \mathrm{~m}$, the lidar is able to resolve wind shear, which has an internationally recognized length scale between $400 \mathrm{~m}$ and $4 \mathrm{~km}$ (Fujita 1978; Proctor et al. 2000; ICAO 2005).

(iv) Ease of deployment-the lidar system is a compact instrument that can be housed in a rectangular fiberglass equipment shelter with a length of 2-3 m on each side. It is therefore much easier to deploy a lidar system in the airport environment compared with a C-band TDWR with $\sim 8$-m-radius antenna dish. Moreover, unlike the weather radar, it is not an active source of electromagnetic radiation in the frequency range for air navigation signals, and thus interference with other systems at the airport is of lesser concern.

The other factors that were considered in deploying the lidar at HKIA are laser safety and equipment reliability. The lidar operates at $2-\mu \mathrm{m}$ wavelength and has pulse energy of about $2 \mathrm{~mJ}$. From accessible emission limit (AEL) calculations, its laser classification during operational use is Class $1 \mathrm{M}$ according to the latest international standard (IEC 2007). Based on maximum permissible exposure (MPE) calculations, the lidar is found to be safe to unaided eyes, even for a stationary laser beam. However, a stationary laser beam is potentially hazardous to the eyes for optically aided viewing (e.g., using magnifying optics such as binoculars). In this connection, laser safety measures including sector blanking and scan rate interlock are implemented in the lidar in view of its proximity to the airport buildings and residential areas just outside the airport (Fig. 1). In accordance with international practice, a laser safety officer has also been appointed within HKO to ensure the effectiveness of these measures. As regards equipment reliability, the lidar has been found to be able to operate round the clock since 2002, achieving system availability of $98 \%$ or above. So far this lidar system is the unit with the longest operation history in the world. The only occasions that necessitated bringing the system out of service for a few hours or more were the replacements of the scanner (typically once every year, due to wear and tear of the mechanical parts) and the laser transceiver (typically once every three years, due to gradual deterioration of the laser diodes and optical alignment). Biannual overhaul maintenance of the lidar, for example, checking the alignment of the laser transceiver, tuning of the scanner, etc., is also arranged outside the wind shear season.

\section{Performance of lidar}

Performance of the lidar has been studied in terms of its measurable range and the quality of the wind data, which are fundamental to the wind shear detection function.

Data availability of the lidar over the four seasons in a typical year (February 2004 to January 2005) have been studied. Noting that in each season, the data availability is more or less isotropic with respect to the azimuth angle, curves of azimuthally averaged data availability are prepared (Fig. 2). Two commonly used plan position indicator (PPI) scans of the lidar are considered, namely, elevation angles of $1^{\circ}$ and $4.5^{\circ}$, which could represent roughly the wind conditions near the arrival and departure corridors. (Some real-life examples of the $0^{\circ}, 1^{\circ}$, and $4.5^{\circ}$ PPI scans of the lidar are given in Fig. 6, showing the typical coverage areas.)

For the $1^{\circ}$ PPI scans, the curves for the different seasons appear to be quite similar. Data are available for at least $70 \%$ of the time up to about $7.5 \mathrm{~km}[8 \mathrm{~km}$ in springtime (February to April)], providing just sufficient coverage for the arrival corridors. For the $4.5^{\circ} \mathrm{PPI}$ scans, the springtime (February to April) sees less lidar velocity data between 1.5 and $7.5 \mathrm{~km}$ compared to the other three seasons, possibly as a result of the lower cloud-base height in spring. Taking an availability threshold of $70 \%$, the maximum measurement range for the $4.5^{\circ} \mathrm{PPI}$ scans in springtime is about $6 \mathrm{~km}$. This corresponds to a height of about $2200 \mathrm{ft}$ (or $670 \mathrm{~m}$ ) AGL assuming a $6^{\circ}$ glide path, which is sufficient to cover the requirement of low-level wind shear alerting service (up to $1600 \mathrm{ft}$ ).

To ensure the quality of the lidar wind data, a routine comparison with the measurements from the anemom- 

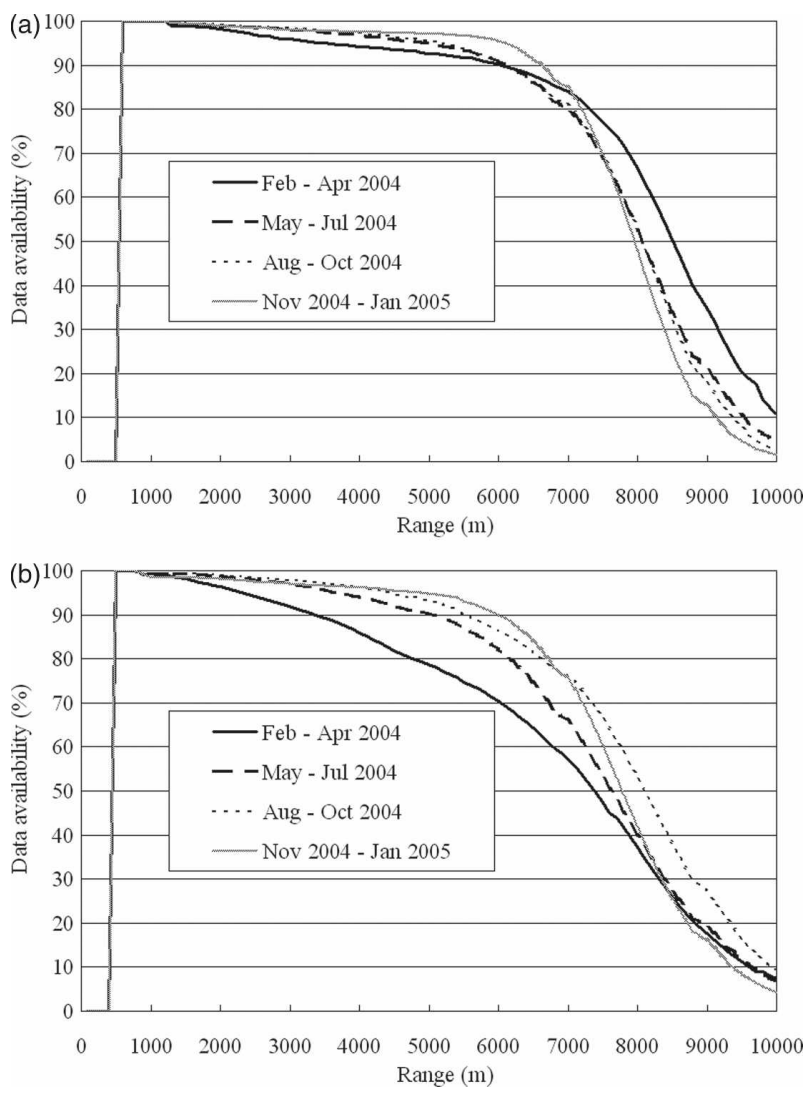

FIG. 2. Data availability curves of (a) $1.0^{\circ}$ PPI scan and (b) $4.5^{\circ}$ PPI scan of the lidar.

eters at HKIA has been carried out. The 1-s average wind data from the cup-vane anemometer R1E [Fig. 1, about $18 \mathrm{~m}$ above mean sea level (MSL) and $880 \mathrm{~m}$ from the lidar], resolved in the radial direction of the laser beam, are compared with the lidar radial velocity measurements at that location from the $0^{\circ}$ PPI scans (50 $\mathrm{m}$ MSL). There are a total number of 110123 data points, and the two sets of measurements are found to be well correlated (upper panel in Fig. 3). The distribution of the number of data points in the various velocity bins is shown in the lower panel of Fig. 3. The bias and RMS difference (lidar minus anemometer) are very small: -0.07 and $1.05 \mathrm{~m} \mathrm{~s}^{-1}$ respectively. The latter value is within the accuracy specifications of the lidar $\left(1 \mathrm{~m} \mathrm{~s}^{-1}\right)$ and the anemometer $\left(0.5 \mathrm{~m} \mathrm{~s}^{-1}\right)$. The lidar instantaneous radial wind measurements from the $1^{\circ}$ PPI scans have also been compared with the 5-min average wind at $170 \mathrm{~m}$ MSL obtained by a $4.5-\mathrm{kHz}$ sodar (sound detection and ranging) (Fig. 1, $7085 \mathrm{~m}$ away from the lidar; the height of the $1^{\circ}$ PPI scan is about $174 \mathrm{~m}$ MSL at the sodar location), again resolved in the radial direction of the laser beam over the sodar. The study period is October 2005 to March 2006. The
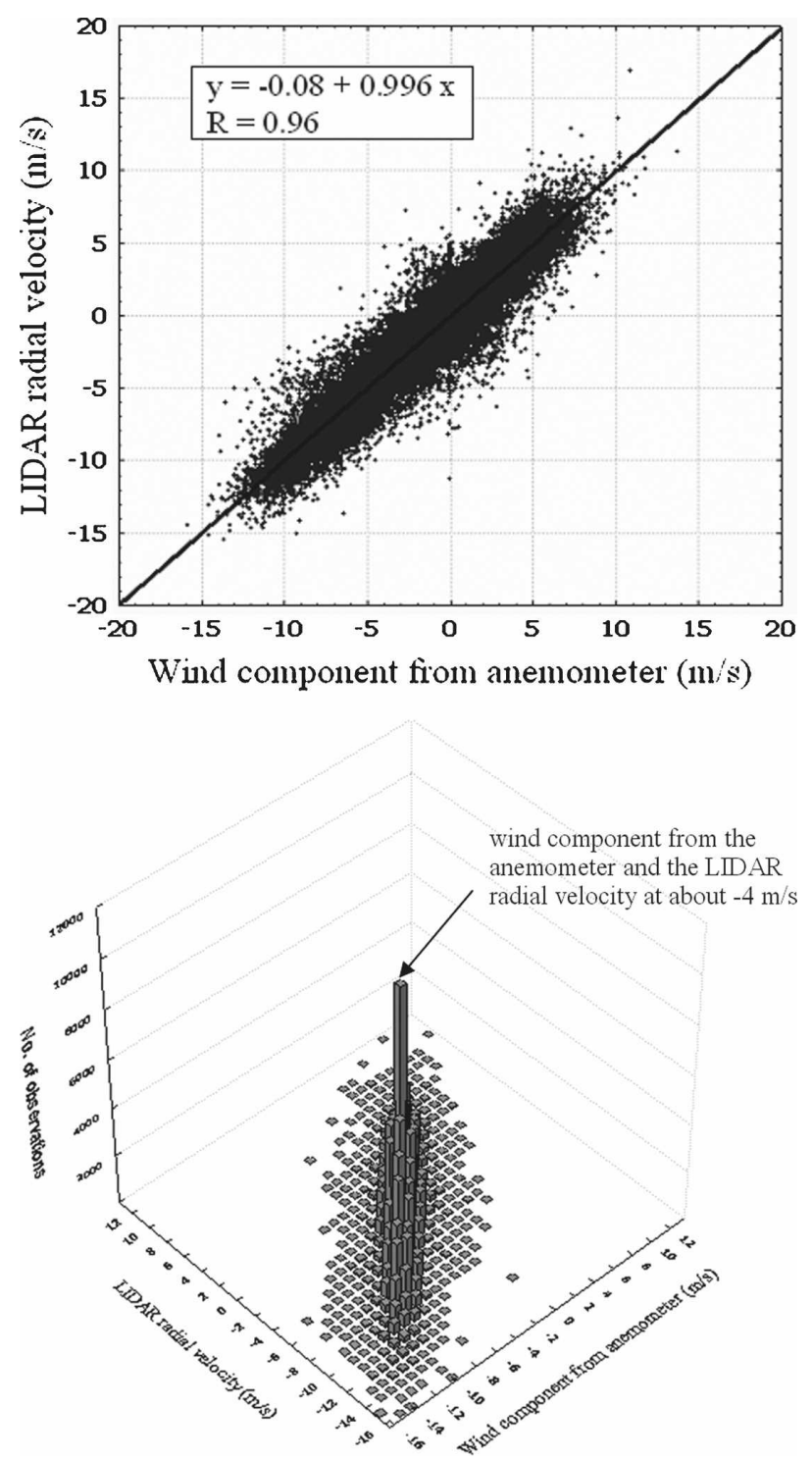

FIG. 3. (top) Comparison between lidar and anemometer data in the period March-November 2004 and (bottom) the distribution of the number of data points in the various velocity bins.

scatterplot of the wind component from sodar and the lidar radial velocity is given in Fig. 4 (upper panel). There are a total number of 18518 data points, and the two sets of measurements are found to be well correlated, but with a slope of about 0.8 much less than unity. This may be due to difference in the sampling volume/ period and the variability of the wind close to the mountains at the location of the sodar. The distribution of the number of data points in the various velocity bins is shown in the lower panel of Fig. 4. The bias is again very small, less than $0.01 \mathrm{~m} \mathrm{~s}^{-1}$. The RMS difference is $1.97 \mathrm{~m} \mathrm{~s}^{-1}$, slightly exceeding the accuracy specifications of lidar $\left(1 \mathrm{~m} \mathrm{~s}^{-1}\right)$ and sodar $\left(0.5 \mathrm{~m} \mathrm{~s}^{-1}\right)$. This may 


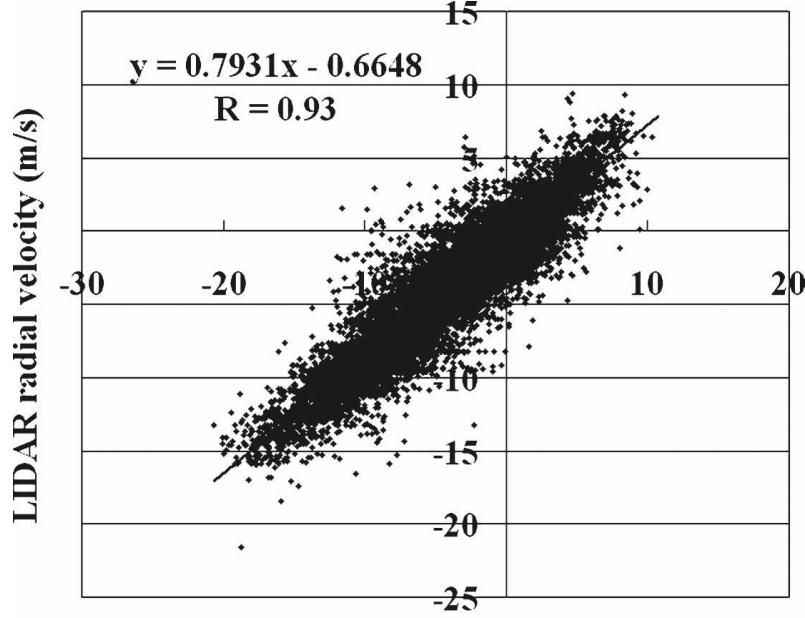

Wind component from SODAR $(\mathrm{m} / \mathrm{s})$

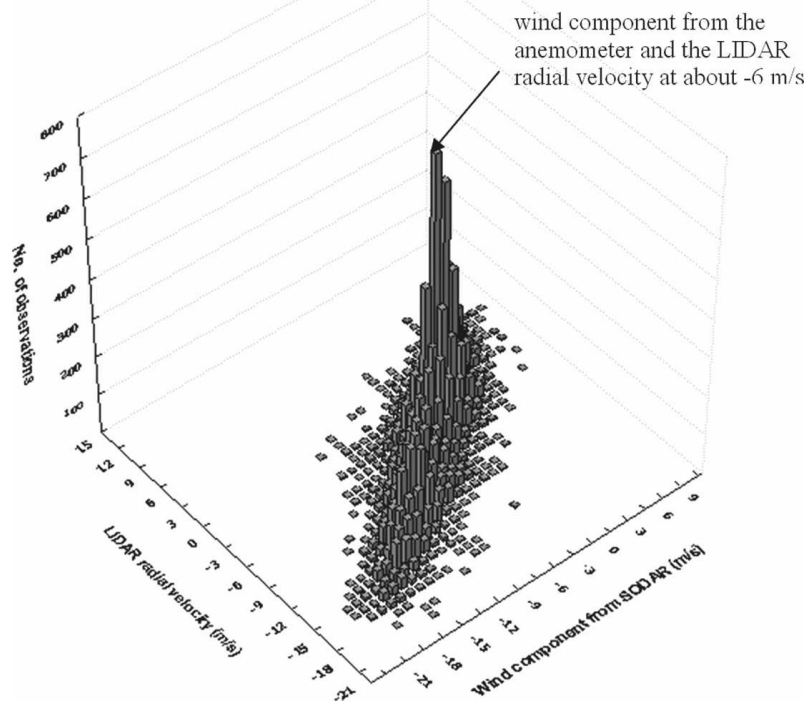

FIG. 4. (top) Comparison between sodar and anemometer data in the period October 2005 to March 2006 and (bottom) the distribution of the number of data points in the various velocity bins.

again be attributable to the difference in sampling volume/period and the variability of the wind.

Accurate measurement of the headwind (with reference to the runway orientation) that the aircraft would experience is fundamental to the successful detection of wind shear. To determine the capability of the lidar in measuring the headwind profile along the glide path, the lidar headwind estimates obtained in the GPScans (which are taken to be the radial velocities directly measured by the lidar without resolving the wind along the runway orientation; see section 7 below) over a commonly used arrival runway corridor 07LA, namely, arriving at the north runway of HKIA from the west,

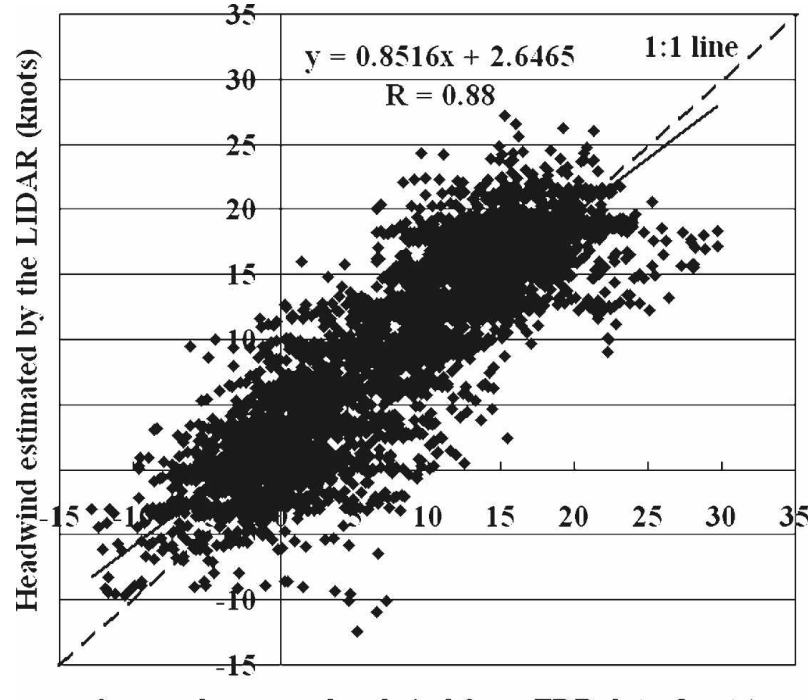

3-second average headwind from FDR data (knots)

FIG. 5. Comparison of the headwind estimate from the second lidar (viz., the radial velocity from the second lidar within $30^{\circ}$ of the runway orientation) and the headwind measured in the QAR data [after processing by the algorithm given in Haverdings (2000)].

are compared with the headwind measurements recorded in the Quick Access Recorder (QAR) onboard commercial transport category aircraft using the same runway corridor. The aircraft data have been processed with a sophisticated QAR wind retrieval algorithm to obtain the wind data at $4 \mathrm{~Hz}$ (Haverdings 2000). Data from 85 flights are used, and the comparison result is shown in Fig. 5 based on the headwind estimates obtained from the second lidar (Fig. 1) because this lidar has better geometry (smaller angle subtended) with respect to the 07LA runway corridor (see section 10). The two datasets are found to be well correlated. The bias is $0.77 \mathrm{~m} \mathrm{~s}^{-1}$ and the RMS difference is $2.1 \mathrm{~m} \mathrm{~s}^{-1}$. Taking into account the accuracy of lidar data $\left(1 \mathrm{~m} \mathrm{~s}^{-1}\right)$, the typical accuracy of aircraft wind data $\left(\sim 0.5-1 \mathrm{~m} \mathrm{~s}^{-1}\right)$ as well as the spatial and temporal differences in the lidar and the flight measurements, the comparison results are considered to be very satisfactory.

\section{Wind shear flow patterns observed by lidar}

When the lidar was first installed in 2002, it was configured to scan in the PPI and range-height indicator (RHI) modes only, following the conventional scan strategies of weather radars. These scans have been used since then and proven to provide a good overview of the wind conditions around HKIA for the weather forecaster to monitor wind shear and to issue/cancel wind shear warning for air traffic control (ATC) and 
pilots. In this section, we describe some typical flow patterns conducive to wind shear occurrence as observed by the lidar.

\section{a. Mountain wake}

The higher mountains on Lantau Island, notably Nei Lak Shan (751 $\mathrm{m}$ ) and Lantau Peak (934 $\mathrm{m}$ ) as shown in Fig. 1, would often generate a wake over the western runway corridors of HKIA under stably stratified easterly flow (Lau and Shun 2000). The wake is clearly discernable in the lidar observations, characterized by generally weaker flow with wind direction rather different from the prevailing direction. Figure 6a shows an example in the morning of 30 March 2005 (Szeto and Chan 2006). Between 0000 and 0300 UTC on that day, there were altogether 15 aircraft reporting significant wind shear on approach from the west.

\section{b. Gap flow}

Under the prevalence of strong winds from the east, southeast-to-southwest sector, accelerated airflow would emerge from the gaps of Lantau Island and affect the runway corridors of HKIA. This could happen in springtime (Shun et al. 2003a) and in tropical cyclone conditions (Shun et al. 2003b). An example in the morning (local time $=\mathrm{UTC}+8 \mathrm{~h}$ ) of 5 March 2003 is shown in Fig. 6b. Five jets associated with the gap flows emerging from the various valleys of Lantau were observed by the lidar. On that day, 26 pilot reports of significant wind shear were received.

\section{c. Mountain wave}

In a stable boundary layer in springtime, a stationary mountain wave train is sometimes observed to stay over the eastern corridors of HKIA, as revealed in both the radial velocity and backscatter imageries of the lidar (Shun et al. 2004). Figure 6c shows a case in the evening of 3 January 2004. The zigzag pattern in the radial velocity data is believed to be associated with lee wave downstream of Lo Fu Tau (Fig. 1), a hill with a height of $465 \mathrm{~m}$ on Lantau Island. An aircraft reported the encounter of significant headwind loss in this case as it departed from the south runway of HKIA toward the east.

\section{d. Sea breeze}

As the sea breeze always forms in fine weather, the movement and structure of the sea-breeze front are usually well captured by the lidar. Figure $6 \mathrm{~d}$ gives an example of the setting in of the sea breeze from the west under the prevalence of background easterly wind on 6 March 2005 (Szeto and Chan 2006), which is very typical in winter and spring at HKIA. Six aircraft arriving at the north runway of HKIA from the west reported the encounter of significant headwind gain as they flew from the westerly sea breeze into the background easterly. However, in some cases the aircraft could experience headwind loss in a sea-breeze situation when the glide path cuts across the density current head associated with the front twice. This has also been observed by the lidar and reproduced successfully using high-resolution numerical modeling (Chan and Szeto 2006).

\section{e. Hydraulic jump and vortex}

Jumplike features are sometimes revealed by the lidar RHI scans toward Lantau when the airflow comes from behind the mountains (Shun et al. 2003a; Banta et al. 2006). They occur most frequently in springtime. Temperature inversion and a low-level jet within the boundary layer were found to be related to their formation downwind of Lantau (Chan and Shun 2006). One example is given in Fig. 6e, which was analyzed in detail by Shun et al. (2003a). A jumplike feature is revealed by the lidar downwind of Lo Fu Tau. Reverse flows are found below and above the jump. The reverse flow below the jump appeared to be associated with a horizontal recirculation vortex near the ground from the lidar low-elevation-angle PPI scans (Fig. 6f). Smaller pockets of reverse flow were also observed to be shed downwind from this region in the subsequent lidar scans (not shown). In another study by Chan and Shun (2005) using a shallow-water model for a stably stratified easterly flow, shedding of vortices downwind of Lo Fu Tau in close agreement with the lidar observations was successfully simulated. The shedding period was found to be of the order of $10 \mathrm{~min}$. Wind shear is to be expected if these features intersect the flight paths. Furthermore, Shun et al. (2003b) found that even in near-neutral conditions during the passage of tropical cyclones, vortex shedding with period of 5-8 min could take place downwind of Lantau in a strong southwesterly airstream.

Considering the complicated three-dimensional flow structures bringing wind shear downwind of Lantau, and the intermittency exhibited by vortex shedding, it becomes apparent that accurate warning of the terraininduced wind shear for HKIA faces a unique challenge due to its transient and sporadic nature.

\section{Transient and sporadic nature of wind shear}

As illustrated in section 5 above, with high spatial resolution $(100 \mathrm{~m})$ and fast data updates $(\sim 1-2 \mathrm{~min})$, 
(a)

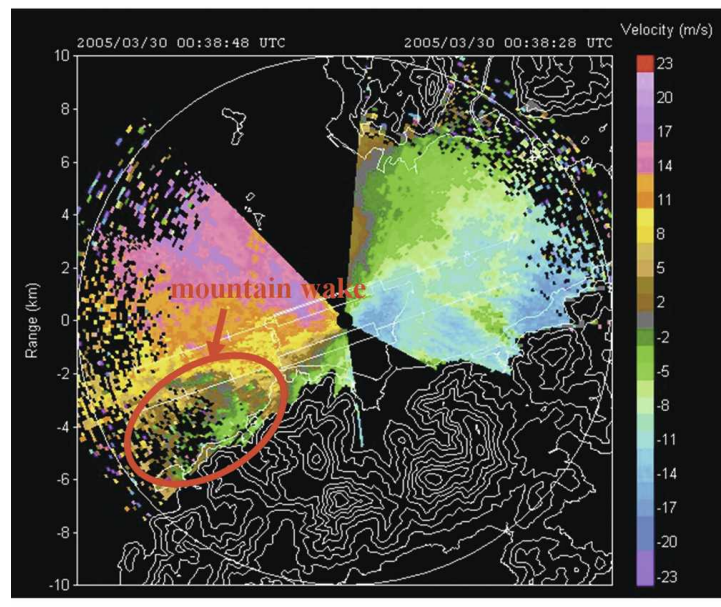

(c)

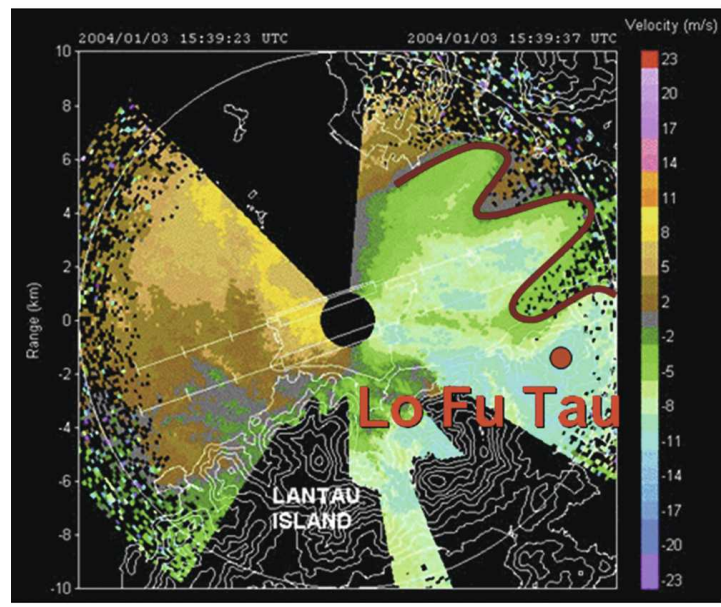

(e)

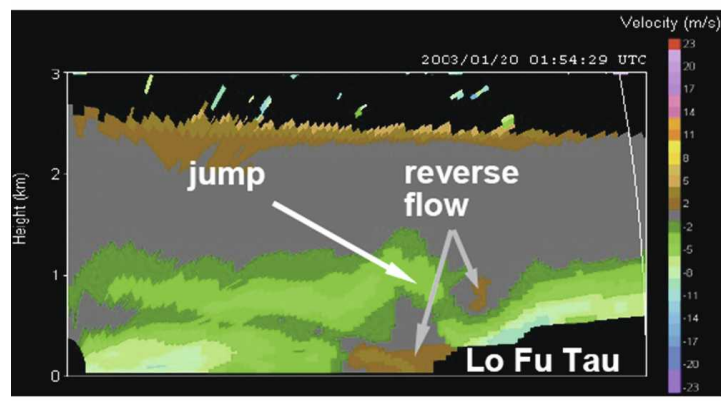

(b)

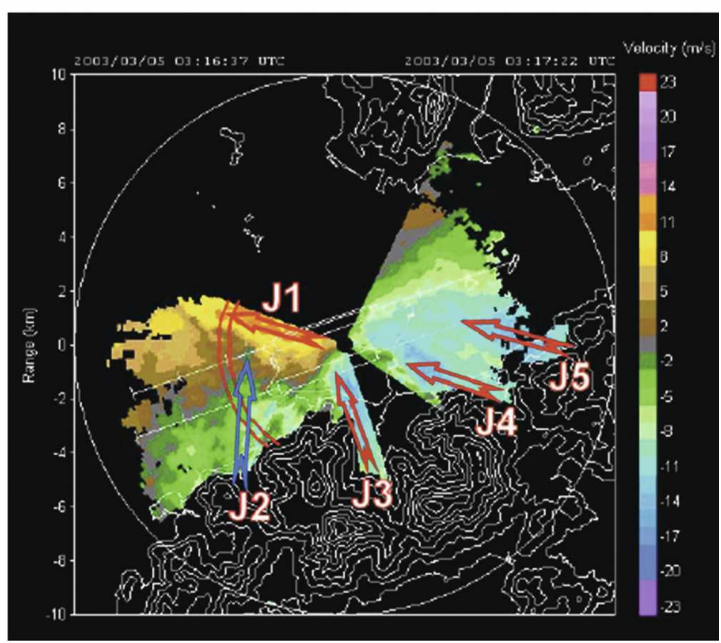

(d)

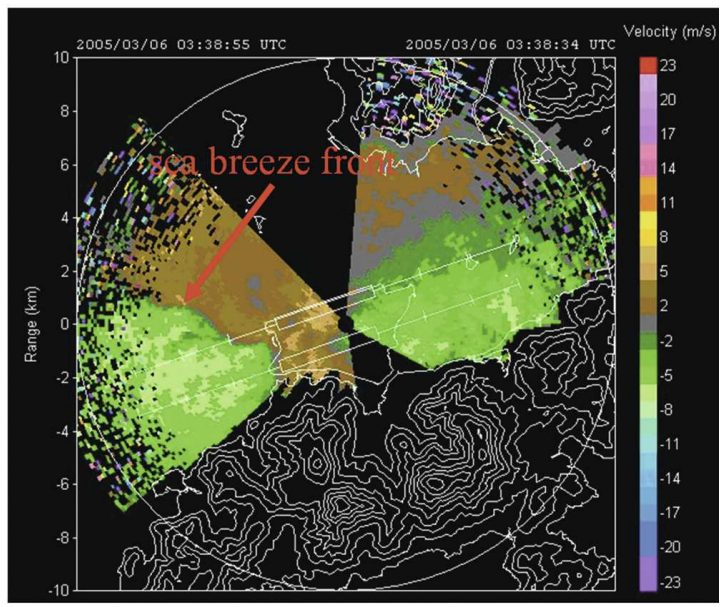

(f)

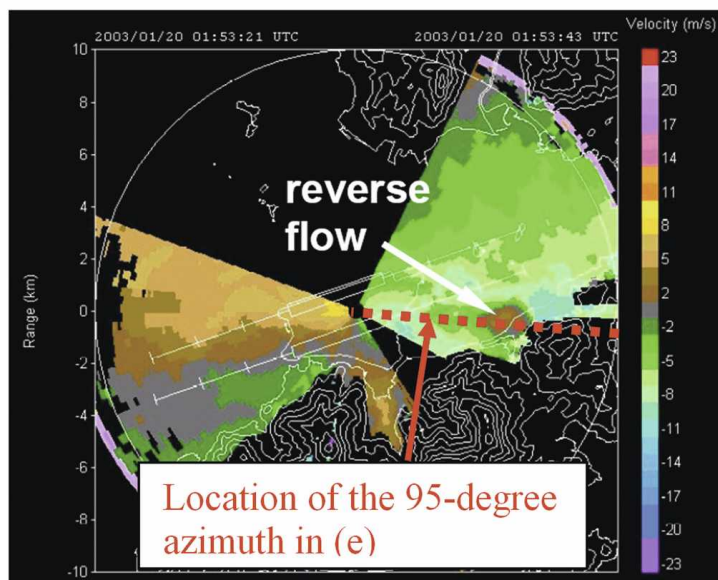

FIG. 6. Wind shear flow patterns observed by the lidar at HKIA. (a) Mountain wake observed in the $1^{\circ}$ PPI scan of the lidar at 0038 UTC 30 Mar 2005. (b) Accelerated gap flow (labeled "J") observed in the $1^{\circ}$ PPI scan of the lidar at 0316 UTC 5 Mar 2003. (c) Mountain wave observed in the $4.5^{\circ}$ PPI scan of the lidar at 1539 UTC 3 Jan 2003. (d) Westerly sea breeze against the background easterly winds as observed in the $0^{\circ}$ PPI scan of the lidar at 0338 UTC 6 Mar 2005. (e) Hydraulic jump of cross-mountain airflow observed in the RHI scan of the lidar at $95^{\circ}$ azimuth [see (f)] at 0154 UTC 20 Jan 2003. (f) Reverse flow of a recirculation vortex underneath the hydraulic jump in (e) in the $1^{\circ}$ PPI scan of the lidar at 0153 UTC 20 Jan 2003. 


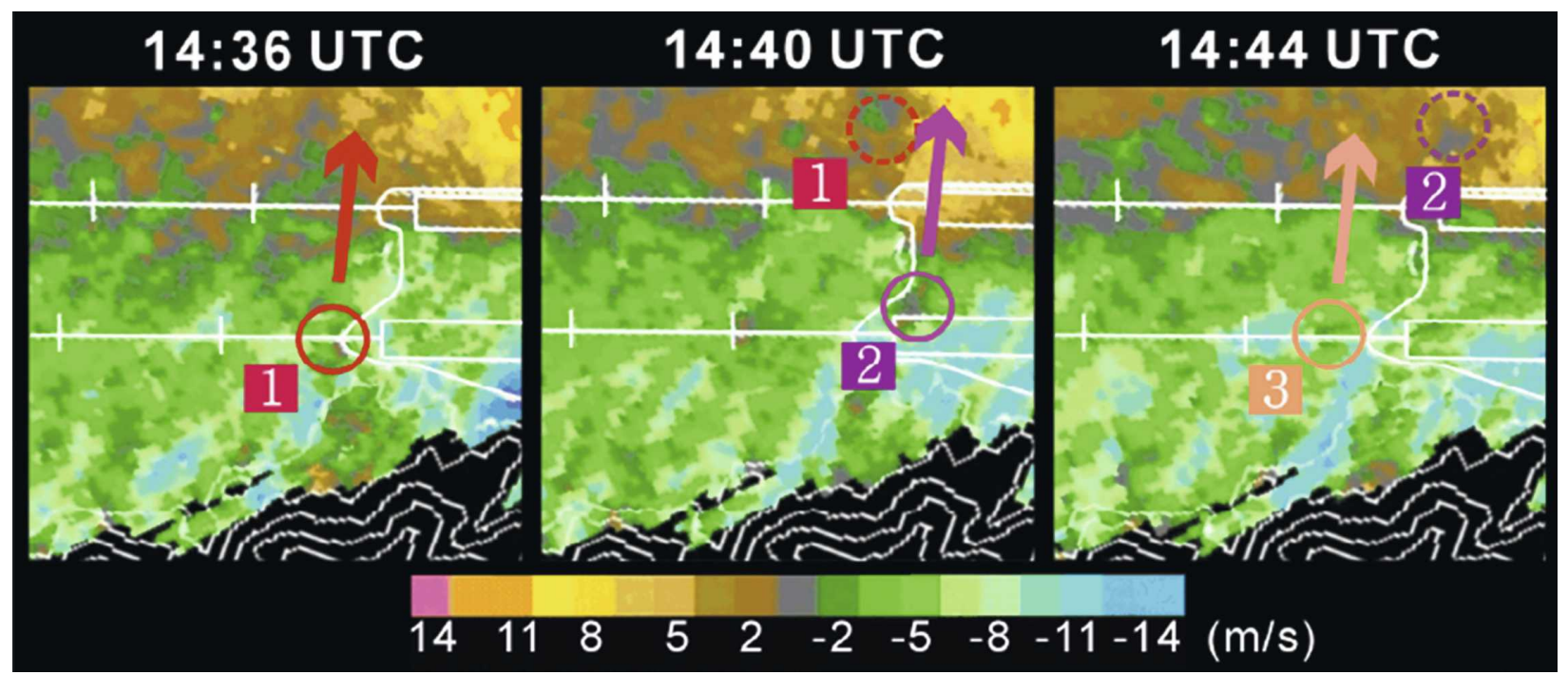

FIG. 7. The $1^{\circ}$ PPI scans of the lidar on 30 Aug 2004. The cool (warm) colors represent winds toward (away) from the lidar (see scale at the bottom). The arrows indicate the movement of the wind shear features marked by circles within the subsequent 4 min.

the lidar is able to reveal many salient features of terrain-induced airflow disturbances. In many cases observed by the lidar, and also confirmed by aircraft data, such disturbances are generally small-sized, with horizontal length scale of several hundred meters or less. Such a case occurred on 30 August 2004 (Fig. 7). On that day, an area of low pressure over the southwestern part of China brought strong southerly winds to Hong Kong. As shown from the $1^{\circ}$ elevation PPI scans of the lidar, there were areas of reverse flow to the west of HKIA as embedded in the background southerly wind, generated from disruption of the southerly airflow by the hills on Lantau Island.

Advected by the strong background wind, these airflow disturbances only affect a particular runway corridor intermittently due to their small size and high advection speed across the corridor. For example, an airflow disturbance with horizontal size of $600 \mathrm{~m}$ advected by a wind of $15 \mathrm{~m} \mathrm{~s}^{-1}$ would only intersect the flight path in $40 \mathrm{~s}$. Furthermore, as shown in Fig. 7, the life cycle of these small-scale terrain-induced disturbances-emergence from the terrain, advection across the runway corridors, and dissipation-could be typically a few minutes. For an aircraft on approach (with typical speed of $75 \mathrm{~m} \mathrm{~s}^{-1}$ ) traversing these small-scale features, the change in headwind would only last $10 \mathrm{~s}$ or less.

Because of the above characteristics of terraininduced airflow disturbances, the wind shear experienced by aircraft is transient and sporadic. It is common for an aircraft to encounter significant wind shear but with the preceding and the following aircraft reporting no wind shear or events of different impact (e.g., headwind loss versus headwind gain). A successful wind shear warning service provided by the lidar would therefore require the following:

1) general overview of the winds in the airport area, whereby the weather forecaster could monitor the presence of wind shear and issue/cancel the wind shear warning for ATC and pilots. This is achieved through the conventional PPI and RHI scans of the lidar; and

2) zoom-in of the wind fluctuations along the individual glide paths, whereby automatic wind shear alerts could be issued on a minute-to-minute basis for relay to aircraft via ATC (or data link to the aircraft cockpit in the future). This is achieved by a new kind of scan strategy devised by HKO to measure the winds along the glide paths-the GPScans (section 7).

\section{Glide path scan}

The concept behind the design of the GPScans is to measure the headwind along the individual glide paths of the airport so that the wind shear to be encountered by the aircraft flying along the glide path could be determined. In making the GPScans, the laser beam of the lidar is configured to slide along the glide paths, as shown schematically in Fig. 8. Also shown in this figure are the scanning areas of GPScans over the final $3 \mathrm{n} \mathrm{mi}$ along the arrival runway corridors of HKIA. The duration of a GPScan over a runway corridor ranges be- 

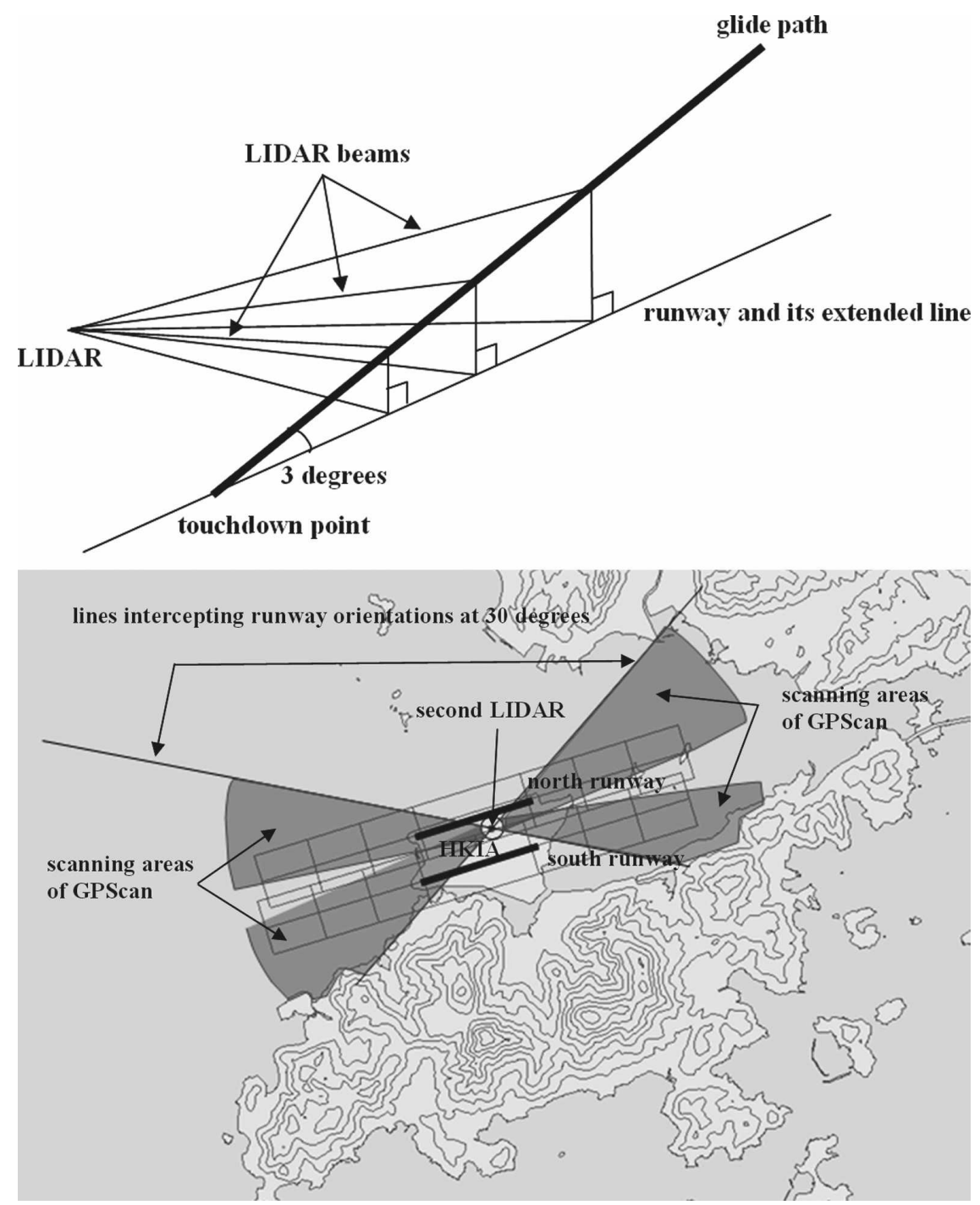

FIG. 8. (top) Schematic diagram of a GPScan of the lidar. (bottom) The scanning areas of GPScans over the final $3 \mathrm{n}$ mi along the arrival runway corridors of HKIA. The second lidar has a perpendicular distance of $480 \mathrm{~m}$ to the north runway and $1040 \mathrm{~m}$ to the south runway of HKIA.

tween 4 and $15 \mathrm{~s}$. The slower scans are used for ongoing studies on using GPScan data to detect low-level turbulence to be encountered by the aircraft, which will not be covered in this paper.

The GPScan concept is particularly applicable to aircraft on approach, which closely follow the $3^{\circ}$ glide slope in the final $3 \mathrm{n}$ mi prior to touchdown on the runway. The GPScans for the arrival corridors are therefore made toward the $3^{\circ}$ glide paths ending at the touchdown points on the runways. The design of the GPScan is not so straightforward for aircraft on departure since the aircraft could adopt glide paths with different elevation angles and originating points (i.e., the points of rotation) on the runway. For simplicity, the
GPScan for the departure corridors are made toward the glide paths with $6^{\circ}$ elevation starting from the middle of the runways.

The flexibility in configuring the lidar scan strategy allows concerted azimuth and elevation motions of the lidar scanner so that the laser beam could slide smoothly along the glide paths. Otherwise, the beam would take stepwise jumps, speeding up the wear and tear of the scanner motors. To enable rapid revisit of each glide path (once every couple of minutes), the scanner is configured to rotate at a rate of $15^{\circ}-18^{\circ} \mathrm{s}^{-1}$ between consecutive GPScans, which is close to the maximum allowable scanning speed of the scanner motors $\left(\sim 20^{\circ} \mathrm{s}^{-1}\right)$. 


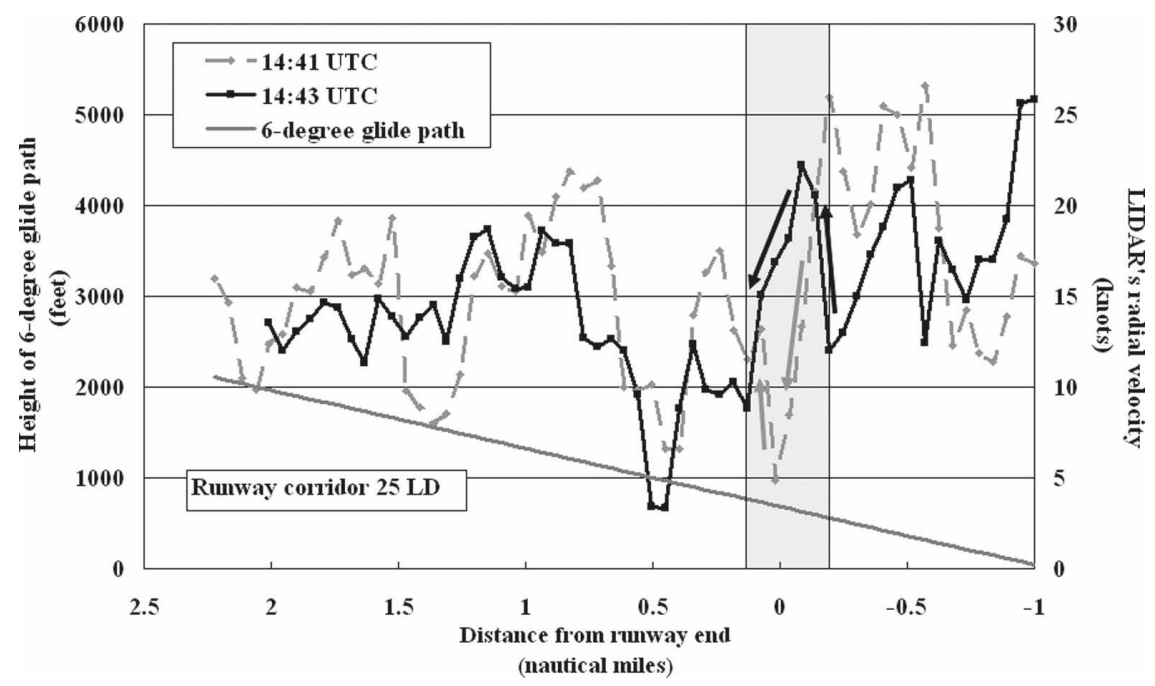

FIG. 9. The headwind profiles of 25LD as measured by the lidar at 1041 and 1043 UTC 30 Aug 2004. Inside the light gray area, the gain/loss sequence in the headwind profile is reversed in this 2-min interval.

From each GPScan, all available lidar radial wind measurements closest to the glide path are collected for constructing the headwind profile-we consider a "tube" within a horizontal distance of $1000 \mathrm{ft}(300 \mathrm{~m})$ and a vertical distance of $200 \mathrm{ft}(60 \mathrm{~m})$ from the glide path. At the location of the first lidar (Fig. 1), the laser beam cuts across the runways at angles depending on the azimuthal angles. An angle threshold of $30^{\circ}$ is currently adopted for the GPScan wind data collection-if the angle of intercept is larger than this threshold, the lidar radial wind data would not be collected for constructing the headwind profile considering the increasing underestimation of the headwind component and the increasing contribution of crosswind to the radial velocity as this angle increases. With the above constraints, the data points within the "tube" that are closest to the glide path are chosen to construct the headwind profile.

The GPScan headwind profiles so generated are able to vividly illustrate the transient and sporadic nature of terrain-induced wind shear (Chan et al. 2006). Figure 9 shows such an example over the departure corridor $25 \mathrm{LD}$, namely, departing from the south runway of HKIA toward the west: at 1441 UTC 30 August 2004, in the direction of the departure corridor, there was a headwind loss of $20 \mathrm{kt}$ followed by a headwind gain of $10 \mathrm{kt}$ around the runway end (indicated in light gray in Fig. 9). However, the headwind sequence in the same region was found to reverse only 2 min later, with a headwind gain of $10 \mathrm{kt}$ followed by a headwind loss of nearly $15 \mathrm{kt}$. An aircraft departing at 25LD at 1441 UTC on that day reported an encounter of significant wind shear. If the aircraft departed 2 min later, it might have reported no wind shear, or reported wind shear with a different magnitude and/or sequence of events. In this case, as in many other cases of terrain-induced wind shear at HKIA, both headwind gain and loss coexisted along the glide path, as revealed by the GPScan headwind profile in Fig. 9.

\section{Lidar Windshear Alerting System (LIWAS)}

For automatic detection and alerting of wind shear along the glide paths, significant changes must be identified from the GPScan headwind profiles using a computer algorithm. Following international practice, the wind shear alerts will also need to be provided to aircraft via ATC using concise alphanumeric messages, indicating the location and magnitude of the most significant change of headwind along the runway corridor concerned, for example, "07LA WSA -25K 2MF" (viz., wind shear alert over 07LA runway corridor, the most significant event being headwind loss of $25 \mathrm{kt}$, with first encounter of the wind shear event at $2 \mathrm{n} \mathrm{mi}$ final).

The basic function of the wind shear algorithm is to look for a sustained change of the headwind, known as a wind shear ramp (Fig. 10). The lidar data are first quality controlled to remove the "spikes" in the headwind profiles arising from clutters, as well as small-scale wind fluctuations associated with jet exhaust or wake turbulence of aircraft. Such outliers could be detected by mimicking visual inspection to compare each piece of radial velocity with the data points around, and re- 


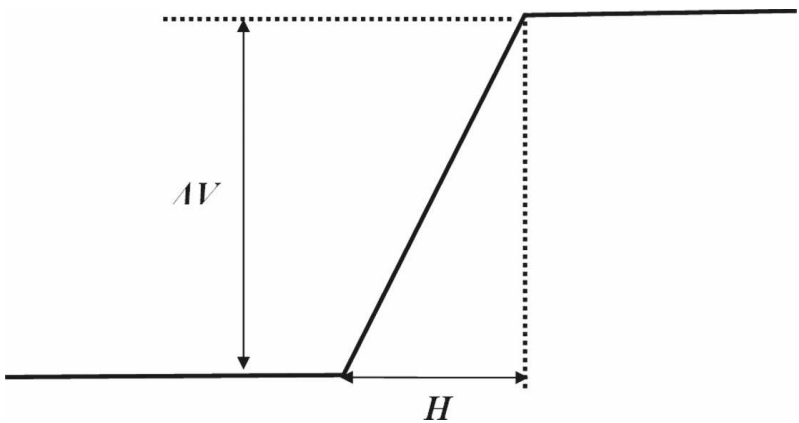

FIG. 10. Schematic diagram of a wind shear ramp with headwind change $\Delta V$ over ramp length $H$.

placed by a median-filtered value if the difference between them is larger than a predefined threshold. The threshold is determined from the frequency distribution of velocity difference between adjacent range/ azimuthal gates of the lidar over a long period of time. Data quality control is kept to a minimum in order not to smooth out the genuine wind fluctuations of the atmosphere.

As discussed in section 7, the lidar radial velocities along a glide path are put together to form a headwind profile. The velocity difference between adjacent data points along the headwind profile is then calculated to construct a velocity increment profile. Following Jones and Haynes (1984), wind shear ramps are successively detected from the velocity increment profile with the ramp lengths increased by a factor of 2 , namely, 400 , $800, \ldots, 6400 \mathrm{~m}$. Before the detection of a ramp length at the $n$th step, say $H_{n}$, the velocity increment profile is smoothed to filter out the velocity fluctuations with spatial scales less than $H_{n}$ so that only ramp lengths in the order of $H_{n}$ would be detected. Following a suggestion of Haynes (1980), the headwind profile is artificially lengthened using the last valid velocity data available at each end. The lengthening is made for each end up to about the original length of the headwind profile. In this way, the data point removal in each smoothing process would only take away the artificial, "constant" velocities and do not affect the genuine wind data.

The wind shear ramps are the peaks and troughs in the smoothed velocity increment profile. They are detected by comparing each data point of this profile with the neighboring points on its both sides. The ramp determined in this way (the "original" ramp) is further adjusted by expansion or contraction in order to capture the full strength of the headwind change across the adjusted ramp. This adjustment is required because, in the smoothing process to determine wind shear ramps of longer lengths, the peaks and troughs of the smoothed velocity increment profile may not corre-

\section{(a)}

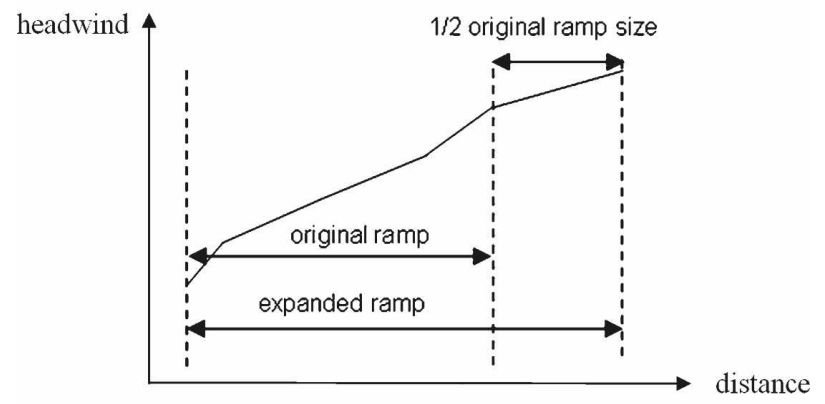

(b)

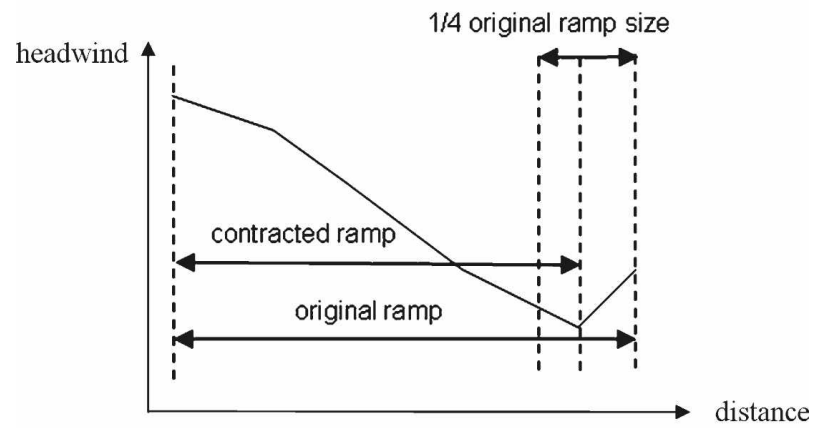

(c)

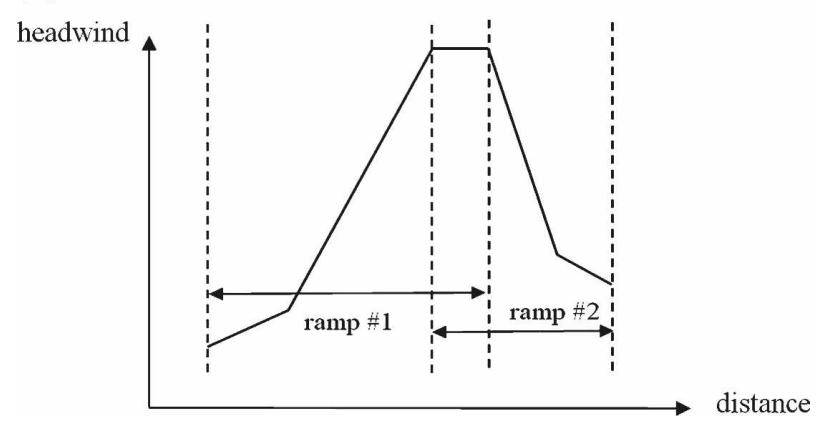

FIG. 11. Schematic diagrams of (a) ramp expansion and (b) ramp contraction. An example of overlapping ramps is given in (c).

spond to the peaks and troughs of the original, unsmoothed profile. The expansion or contraction adjustments would then help locate the maximum wind changes associated with the longer ramps. For instance, for a ramp of headwind gain, if the velocity continues to rise after the original ramp, the ramp length is expanded to cover the velocity increasing part until the velocity starts to drop or half of the original ramp length is reached, whichever is shorter (Fig. 11a). On the other hand, for a ramp of headwind loss, if velocity increases at the final portion of the ramp, the ramp length is contracted until the velocity increasing part is passed or a quarter of the original ramp length is reached, whichever is smaller (Fig. 11b). 
In general, a headwind profile contains more than one wind shear ramp. The detected ramps are prioritized according to the severity factor $S$ proposed in Woodfield and Woods (1983) so as to determine the most significant event along the glide path:

$$
S=\left(\frac{d V}{d t}\right)\left(\frac{\Delta V}{V_{\text {app }}}\right)^{2}=\left(\frac{\Delta V}{H^{1 / 3}}\right)^{3} / V_{\text {app }}
$$

where $d V / d t$ is the rate of change of wind speed (along the glide path), $\Delta V$ is the total change of wind (along the glide path), $V_{\text {app }}$ is the normal approach speed of the aircraft, and $H$ is the ramp length. Thus, the primary parameter turned out to be the normalized wind shear value $\Delta V / H^{1 / 3}$. A similar conclusion was drawn in Jones and Haynes (1984) based on analysis of flight data. LIWAS also adopts the same normalized wind shear value in prioritizing the wind shear ramps detected from a headwind profile. For overlapping ramps (schematic diagram of an example in Fig. 11c), only the one with the highest normalized wind shear value would be retained and the others would be removed.

LIWAS generates a wind shear alert automatically when a wind shear ramp with $\Delta V$ exceeding the alert threshold is detected. Following the internationally adopted wind shear alerting threshold, the LIWAS alert threshold should be set as $15 \mathrm{kt}$. However, since the lidar beam intercepts the runways at HKIA at an angle, only a component of the actual headwind experienced by the aircraft could be measured. This geometrical factor is more significant for ranges near the lidar, especially near the runway thresholds and touchdown zones. Based on studies of the data in 2004 and 2005, the alert threshold is chosen to be $14 \mathrm{kt}$.

The LIWAS alert is ingested into Windshear and Turbulence Warning System (WTWS) operated by HKO to provide wind shear alerts to ATC for relay via voice communications to the pilots. WTWS also integrates alerts from the other wind shear detection systems, including TDWR and the anemometer-based algorithm. The integration is based on a prioritization scheme that considers the significance of the event and credibility of the system issuing the alert (HKO and IFALPA 2005). After integration, one single wind shear alert is generated for each runway corridor.

\section{Performance of LIWAS}

LIWAS was put into operation for the four arrival corridors in December 2005. The performance of LIWAS alerts is examined using three metrics: hit rate (a hit is a pilot report of significant wind shear that has been successfully captured by a wind shear alert), alert
TABLE 1. (a) Performance statistics of LIWAS for the first lidar in the spring of 2006 and 2007 over the most commonly used arrival runway corridor in this season, viz., the 07LA runway corridor. Period of study: 1 Jan to 30 Apr 2006 and 2007 (excluding those dates when data from the first lidar were not available). (b) Definitions of $a, b, c$, and $d$ for the contingency table.

(a)

No. of wind shear reports 358

No. of hits $\quad 272$

No. of null reports $\quad 189$

No. of null reports matched with alerts $\quad 139$

Alert duration (min) 17891

Hit rate* $\quad 76 \%$

Alert duration per hit (min) $\quad 65.8$

False alarm rate* $\quad 33.8 \%$

* With reference to the following contingency table, hit rate $=$ $a /(a+b)$; false alarm rate $=c /(a+c)$.

(b)

\begin{tabular}{|c|c|c|c|}
\hline & & \multicolumn{2}{|c|}{ Wind shear alert } \\
\hline & & $\begin{array}{l}\text { Y (alert } \\
\text { issued) }\end{array}$ & $\begin{array}{l}\mathrm{N} \text { (alert not } \\
\text { issued) }\end{array}$ \\
\hline \multirow[t]{2}{*}{ Pilot report } & $\begin{array}{l}\text { Y (significant wind } \\
\text { shear) }\end{array}$ & $a$ & $b$ \\
\hline & $\begin{array}{l}\mathrm{N} \text { (null wind shear } \\
\text { or wind shear less } \\
\text { than } 15 \mathrm{kt} \text { ) }\end{array}$ & $c$ & $d$ \\
\hline
\end{tabular}

duration per hit (expressed as the total alert duration divided by the number of wind shear hits), and false alarm rate (a false alarm is a pilot report of encountering null wind shear or wind shear with a magnitude less than $15 \mathrm{kt}$ that has been matched with a wind shear alert). The mathematical formulas for calculating hit rate and false alarm rate are given in the explanatory note of Table 1 for ease of reference. The majority of pilot reports are received in springtime. The performance of LIWAS alerts in the spring of 2006 and 2007 over the most frequently used arrival corridor 07LA is summarized in Table 1. The hit rate of the wind shear alerts reaches a relatively high level of $76 \%$ and the alert duration per hit is just about $66 \mathrm{~min}$. The remaining $24 \%$ of the undetected wind shear reports are believed to be related to (i) the transient and sporadic nature of the wind shear versus the limitation in the revisit time of the GPScan over a particular runway corridor; (ii) different perception of the shear effects on the aircraft by the pilots (see discussion below); and (iii) presence of weather (e.g., low clouds) that limited the lidar range.

The false alarm rate in the spring of 2006 and 2007 for $07 \mathrm{LA}$ was about $34 \%$. The 182 pilot reports in 2007 , including the false alarm cases, are analyzed using the Woodfield diagram (Woodfield 1994) (Fig. 12), that is, 


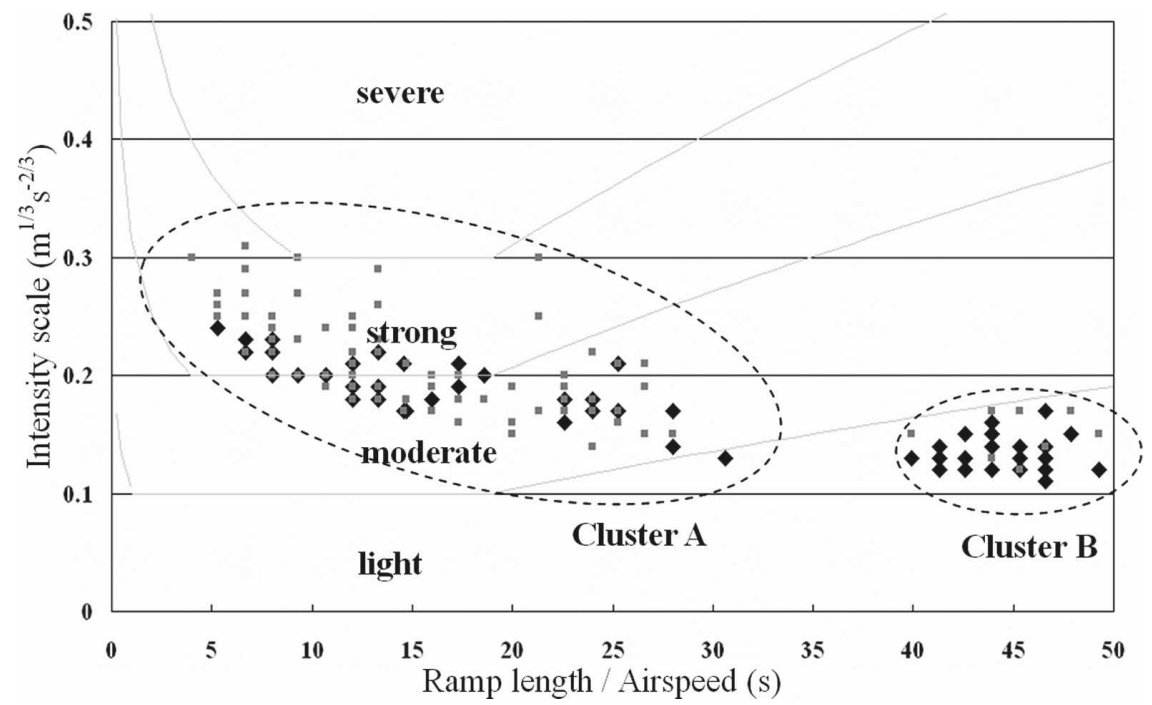

FIG. 12. Wind shear intensity - ramp length diagram for wind shear reports (gray square dots) and null reports (black spades) over 07LA in the period 14 Feb to 31 May 2007 based on wind shear ramps detected by the first lidar (the plot based on wind shear ramps of the second lidar is similar).

the wind shear severity factor $(S)$ versus the ramp length $(H)$ assuming a typical approach speed $\left(V_{\text {app }}\right)$ of $75 \mathrm{~m} \mathrm{~s}^{-1}$. The wind shear ramps are taken from the lidar headwind profiles at the times of the pilot wind shear reports. It turns out that there are two "clusters" of pilot reports in the diagram. One cluster (cluster A) is mainly composed of significant wind shear events as reported by the pilots. They have shorter ramp length (less than $3 \mathrm{~km}$ ) and stronger severity, and are thus concentrated in the left-hand side of the diagram, with the wind shear category considered as "moderate" or "strong" following the classification of Woodfield (1994). The other cluster (cluster B) is largely made up of the false alarm cases. The data points have longer ramp length (all larger than $3 \mathrm{~km}$ ) concentrated in the right-hand side of the diagram with the wind shear category considered as "light" following Woodfield's classification. It appears that a ramp length of $3 \mathrm{~km}$ may be adopted as the threshold delineating significant and nonsignificant wind shear events. However, since the present study is based on limited data from the spring season of one year only, more data would need to be collected to confirm the threshold. When this is achieved, the internationally recognized length scale of $400 \mathrm{~m}$ to $4 \mathrm{~km}$ for wind shear (Fujita 1978; Proctor et al. 2000; ICAO 2005) would need to be revised.

In Fig. 12, while cluster $\mathrm{A}$ is mainly composed of significant wind shear events as reported by pilots, there are still many false alarm cases in this cluster. Similarly, there are also a number of significant wind shear events in cluster B. In this connection, we look at the GPScan headwind profiles and the corresponding pilot reports for a number of such cases and find that similar wind shear events could be perceived very differently by different pilots. Two examples are given in Fig. 13 for illustration. In the first case (Fig. 13a), the lidar's headwind profiles for runway 07LA depict relatively short ramp length $(\sim 1 \mathrm{~km})$ with a headwind gain of $15 \mathrm{kt}$. For the earlier ramp, there was a pilot report (A333) of 20-kt headwind gain below $1000 \mathrm{ft}$ over runway 07LA, consistent with lidar observations. However, for the latter ramp, an aircraft using runway 07LA (B744) gave a wind shear report of 10-kt headwind gain only. Since the magnitude of the reported wind shear is less than $15 \mathrm{kt}$, this latter report is taken to be a null report. The second case (Fig. 13b) refers to relatively gentle wind shear ramps (3-4 km) with headwind gain of $16-18 \mathrm{kt}$ that were separated by $3 \mathrm{~min}$. At the time of the earlier ramp, a pilot (B744) reported encountering wind shear with headwind gain of only $10 \mathrm{kt}$ at $800 \mathrm{ft}$. At the latter time, an aircraft (A333) had to conduct a missed approach due to encountering wind shear at $400 \mathrm{ft}$.

From our discussions with pilots, we understand that they do not yet have direct display of headwind information at the cockpit. As such, different pilots might have different practices in reporting the wind shear and estimating the shear magnitude. For example, they might refer to the change of airspeed shown on the airspeed indicator, or the trend arrow shown on the airspeed indicator, or the wind vector display, to report the wind shear, including the magnitude. Nevertheless, 
(a)

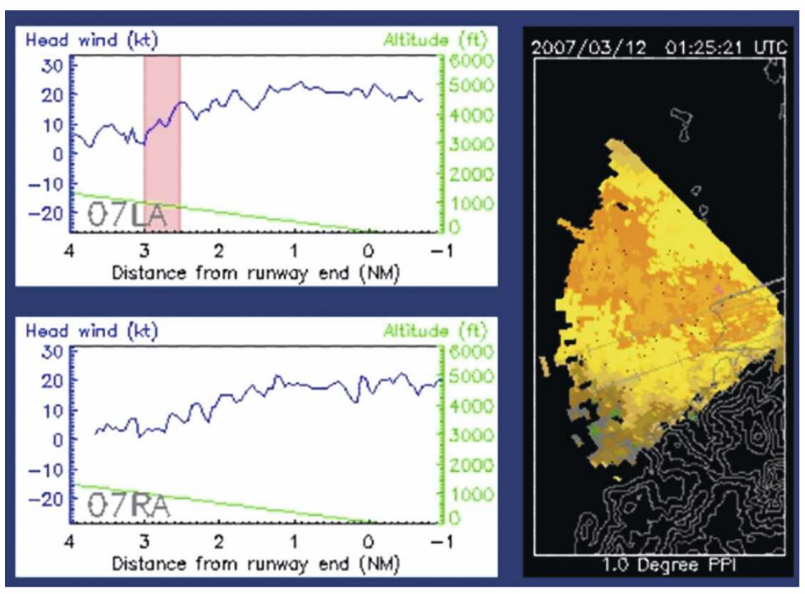

01:31 UTC, 12 March 2007

LIWAS alert (07LA): +15 knots, 3 nm

Pilot report (07LA): below +20 knots, 1000 feet, Pilot report (07LA): +10 knots, 300 feet, B744

\section{A333}

(b)
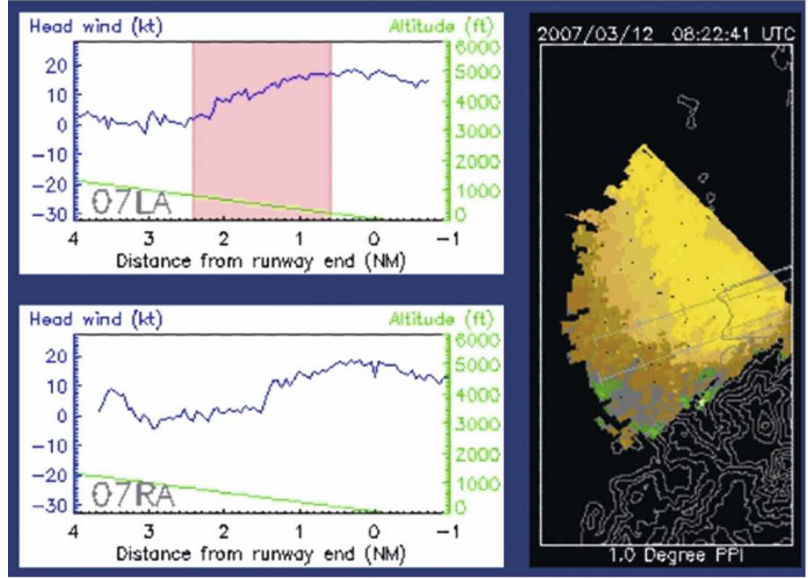

08:25 UTC, 12 March 2007

LIWAS alert (07LA): +16 knots, $3 \mathrm{~nm}$

Pilot report (07LA): +10 knots, 800 feet, B744

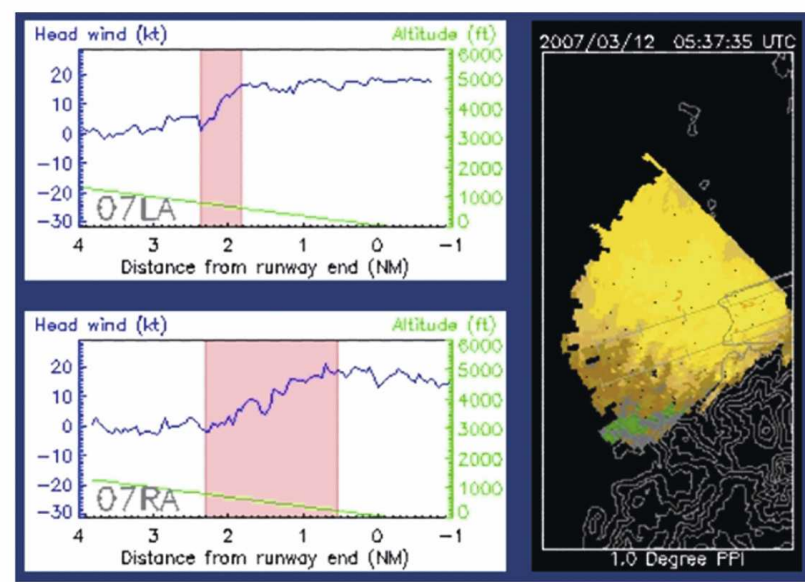

05:39 UTC, 12 March 2007

LIWAS alert (07LA): +15 knots, 3 nm

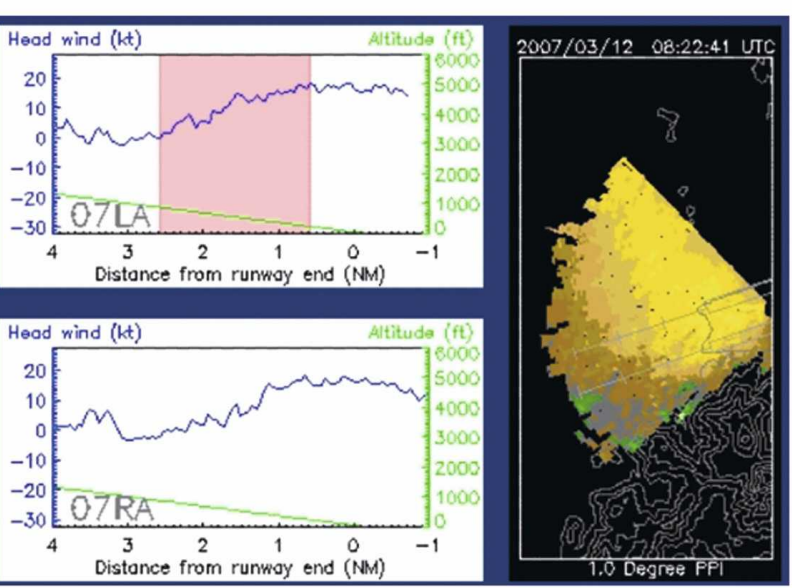

08:28 UTC, 12 March 2007

LIWAS alert (07LA): +18 knots, 3 nm

Pilot report (07LA): missed approach due to

windshear, 400 feet, $\mathrm{A} 333$

FIG. 13. Similar wind shear ramps but with very different pilot reports of wind shear encounter: (a) sharp wind changes at 0131 and 0539 UTC 12 Mar 2007 and (b) gentle wind changes at 0825 and 0828 UTC 12 Mar 2007.

due to their availability and official status in the aviation community, the pilot reports collected by ATC are still used as "sky truth" in our evaluation of the performance of wind shear alerting services and tuning of automatic wind shear algorithms.

In comparison with the pilot reports, QAR data from commercial transport category aircraft should be a more objective source of sky truth for performance evaluation and algorithm tuning. While efforts have been made to obtain QAR data at HKIA by contractual arrangements and to process the QAR data using a sophisticated wind retrieval algorithm (Haverdings 2000), not all the wind shear parameters, such as those in Eq. (1), are automatically computed. Nevertheless, a 


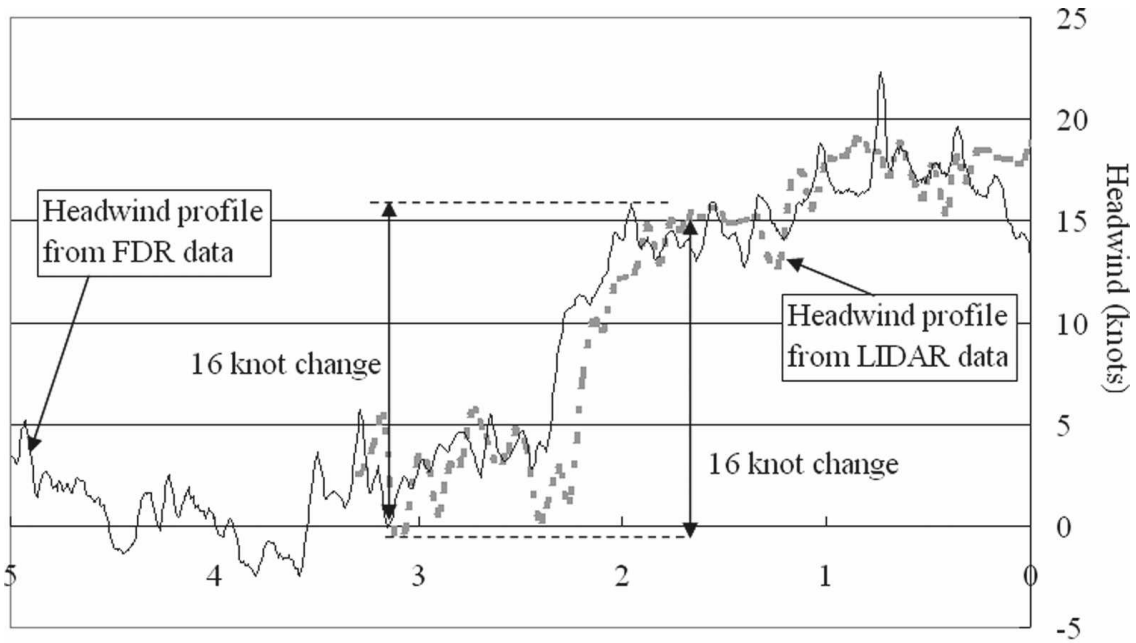

Distance from the runway end (nautical miles)

05:39 UTC, 12 March 2007 (B744)

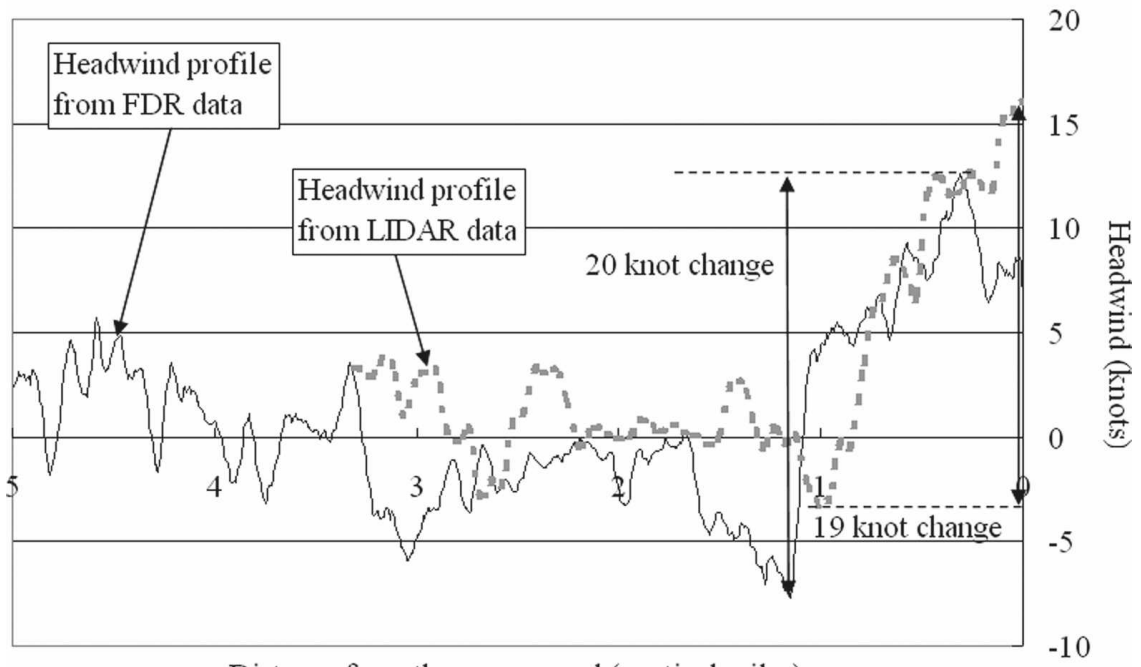

Distance from the runway end (nautical miles)

07:13 UTC, 13 March 2007 (A330)

FIG. 14. (top) The headwind profiles from the QAR data and (bottom) the lidar data in two examples of false alarm cases over 07LA runway corridor in March 2007.

recent study of QAR data for eight cases of the abovementioned false alarm cases with QAR data obtained from the airline revealed that there were significant headwind changes in the majority of the cases. Two examples over the 07LA runway corridor are shown in Fig. 14. In the first case (upper panel in Fig. 14), both the headwind profiles from the QAR data and the second lidar data are generally consistent and show a significant headwind gain of $15 \mathrm{kt}$ from about 3 to $2 \mathrm{n} \mathrm{mi}$ from the runway end. However, the pilot of the aircraft concerned reported headwind gain of only $10 \mathrm{kt}$ at 300 $\mathrm{ft}$, which is taken as a null report because the reported wind shear magnitude is less than $15 \mathrm{kt}$. For the second case (lower panel in Fig. 14), headwind gain in excess of
$15 \mathrm{kt}$ between around $1 \mathrm{n} \mathrm{mi}$ from the runway end and the touchdown zone was depicted in both headwind profiles from the QAR data and the second lidar. The pilot of the aircraft concerned reported headwind loss of only $10 \mathrm{kt}$ at $100 \mathrm{ft}$, which does not seem to be consistent with the headwind profiles. In both cases, the headwind profiles estimated from the radial velocity data of the first and the second lidar are very similar (not shown). It is apparent that the pilot's perception of the effects of the shear could lead to the reporting of these significant wind shear cases as false alarms. The verification results of the LIWAS above, especially the false alarm rates, should be viewed with this perspective. 
TABLE 2. Performance statistics of LIWAS algorithms for the two lidars over the most commonly used arrival runway corridor in the spring of 2007, viz., the 07LA runway corridor. Period of study: 14 Feb to 31 May 2007 (excluding those dates when data from any one of the lidars were not available).

\begin{tabular}{|c|c|c|}
\hline & $\begin{array}{l}\text { First } \\
\text { lidar }\end{array}$ & $\begin{array}{l}\text { Second } \\
\text { lidar }\end{array}$ \\
\hline No. of wind shear reports & \multicolumn{2}{|c|}{134} \\
\hline No. of hits & 96 & 96 \\
\hline No. of null reports & \multicolumn{2}{|c|}{121} \\
\hline No. of null reports matched with alerts & 79 & 72 \\
\hline Alert duration (min) & 7018 & 6220 \\
\hline Hit rate* & $71.6 \%$ & $71.6 \%$ \\
\hline Alert duration per hit $(\mathrm{min})$ & 73.1 & 64.8 \\
\hline False alarm rate* & $45.1 \%$ & $42.9 \%$ \\
\hline
\end{tabular}

* With reference to the following contingency table, hit rate $=$ $a /(a+b) ;$ false alarm rate $=c /(a+c)$.

\section{Runway-specific lidar}

In view of the success of the first lidar, a second lidar was introduced to HKIA in October 2006. It was installed at a location closer to the north runway of HKIA (Fig. 1), to which most of the approaches are made. The second lidar serves as a backup of the first one to ensure the continuous availability of the lidar data for wind shear detection, for example, when the laser transceiver of the first lidar needs to be refurbished and realigned at the factory. If not required to perform the backup function, the second lidar would perform more frequent scans over the north runway (once every minute) in order to better capture the transient and sporadic wind shear associated with terrain disruption of the airflow. Being closer to the north runway, the laser beam from the second lidar is also better aligned with the orientation of this runway, thus better resolving the headwind to be encountered by the arriving aircraft.

The performance of the second lidar in detecting wind shear over the most frequently used arrival runway corridor 07LA in the peak wind shear season in early 2007 is given in Table 2. Compared with the performance statistics of the first lidar in the same period, it could be seen that the second lidar maintains the same hit rate, but the wind shear alerts are more precise with shorter alert duration per hit (decrease by $11 \%$ ) and slightly smaller false alarm rate (drop by $2 \%$ ). The apparently high false alarm rates here should also be viewed with the perspective provided by the QAR data analysis in section 9 .

Encouraged by the success of the second lidar, it is planned to move the first lidar to a site closer to the south runway in late 2007/early 2008 to enhance the wind shear detection over this runway, which is mainly used for departure. The LIWAS algorithm for the departure runway corridors would then be implemented. Wind shear detection for departing flights is considered to be more challenging, as explained in section 7. It may be necessary, for instance, to use RHI scans to cover several possible departing glide paths and search for significant wind shear ramps along all these paths.

\section{Conclusions}

From the experience at HKIA since 2002, the lidar technology is very well suited for the detection and alerting of terrain-induced wind shear under clear-air conditions in an operational airport environment. The lidar system has a high availability level ( $\geqq 98 \%)$ and is able to depict the airflow in the airport area in great detail, both spatially and temporally. For successful alerting of low-level wind shear, HKO makes a number of achievements in the recent years with the lidar, including the design of the GPScans to measure the headwind to be encountered by the aircraft and the development of automatic wind shear detection algorithm based on the headwind profiles. A specific lidar has also been deployed to serve a particular runway (the north runway of HKIA as a start) to enhance wind shear detection with more frequent scans and better alignment of the laser beam with respect to the runway orientation. With all these efforts, the hit rate of wind shear detection by LIWAS on its own reaches a high level of about $76 \%$ (for 07LA runway corridor-the most-used arrival runway corridor in the springtime peak wind shear season).

HKO is developing the automatic lidar-based wind shear detection algorithm for the departure corridors. Apart from wind shear detection, further work will be conducted on the other aviation applications of the lidar, including the automatic alerting of turbulence, visibility monitoring, and synergistic combination with radars to measure the wind field over the airport area in virtually all weather conditions.

Acknowledgments. The authors thank the following colleagues in HKO for their contributions to the lidar project: Dr. C. M. Cheng and Ms. Olivia S. M. Lee for the installation of the first lidar, Mr. B. L. Choy for the development of LIWAS algorithm, Messrs. L. M. Hui, L. K. Yau, and other members of the HKO electronic maintenance team in ensuring the smooth operation of the lidar over the years, and, last but not least, the research assistants/programmers Ms. M. L. Kuo, Messrs. K. K. Lai, S. P. Lui, K. C. Wu, and C. M. Li for developing and maintaining the LIWAS software. Spe- 
cial thanks also go to the pilots and Civil Aviation Department of the Hong Kong Special Administrative Region Government for the provision of wind shear reports, and to Cathay Pacific Airways for the supply of QAR data with agreement of the Hong Kong Aircrew Officers Association (HKAOA).

\section{REFERENCES}

Banta, R. M., L. D. Olivier, and J. M. Intrieri, 1990: Doppler lidar observations of the 9 January 1989 severe downslope windstorm in Boulder, Colorado. Preprints, Fifth Conf. on Mountain Meteorology, Boulder, CO, Amer. Meteor. Soc., 68-69.

-, - and D. H. Levinson, 1993: Evolution of the Monterey Bay sea-breeze layer as observed by pulsed Doppler lidar. $J$. Atmos. Sci., 50, 3959-3982.

- and Coauthors, 2006: Detection and diagnosis of wind shear and turbulence using Doppler LIDAR at Hong Kong International Airport. ESRL/NOAA and HKO Rep., 130 pp.

Biron, P. J., M. A. Isaminger, K. J. Flemming, and A. A. Borho, 1990: A case study of the Claycomo, Mo. microburst on July 30, 1989. Preprints, 16th Conf. on Severe Local Storms, Kananaskis Park, AB, Canada, Amer. Meteor. Soc., 388-392.

Bougeault, P., and Coauthors, 2001: The MAP Special Observing Period. Bull. Amer. Meteor. Soc., 82, 433-462.

Chan, P. W., and C. M. Shun, 2005: Numerical simulation of vortex shedding observed at the Hong Kong International Airport using a shallow water model. Croat. Meteor. J., 40, 27-30.

- , and - 2006: Application of a microwave radiometer to study terrain-induced airflow disturbances at the Hong Kong International Airport. Preprints, 12th Conf. on Mountain Meteorology, Sante Fe, NM, Amer. Meteor. Soc., P2.18.

— of terrain-disrupted airflow and sea breeze (in Chinese with English abstract). Proc. 20th Guangdong - Hong Kong Macao Technical Seminar on Meteorological Science and Technology, Macao, China.

— C. M. Shun, and K. C. Wu, 2006: Operational LIDAR-based system for automatic windshear alerting at the Hong Kong International Airport. Preprints, 12th Conf. on Aviation, Range, and Aerospace Meteorology, Atlanta, GA, Amer. Meteor. Soc., 6.11. [Available online at http://ams.confex.com/ ams/pdfpapers/100601.pdf.]

Fujita, T. T., 1978: Manual of downburst identification for Project NIMROD. University of Chicago, SMRP Research Paper 156, 104 pp.

Hannon, S. M. and J. A. Thompson, 1994: Aircraft wake vortex detection and measurements with pulsed solid-state coherent laser radar. J. Mod. Opt., 41, 2175-2196.

_ H. R. Bagley, and R. K. Bogue, 1999: Airborne Doppler lidar turbulence detection: ACLAIM flight test results. Laser Radar Technology and Applications IV, G. W. Kamerman and Christian Werner, Eds., International Society for Optical Engineering (SPIE Proceedings, Vol. 3707), 234-241.

Haverdings, H., 2000: Updated specification of the WINDGRAD algorithm. NLR TR-2000-63, National Aerospace Laboratory, $68 \mathrm{pp}$.

Haynes, A., 1980: Description of a program developed for the analysis of windshears experienced during aircraft approach to landing. Royal Aircraft Establishment Tech. Memo. FS $321,25 \mathrm{pp}$.

HKO and IFALPA, 2005: Windshear and turbulence in Hong
Kong-Information for pilots. Hong Kong Observatory and International Federation of Air Line Pilots' Associations, 29 pp.

ICAO, 2005: Manual on Low-level Windshear and Turbulence. 1st ed. International Civil Aviation Organization, $222 \mathrm{pp}$.

_ 2007: Meteorological Service for International Air Navigation: Annex 3 to the Convention on International Civil Aviation. 16th ed. International Civil Aviation Organization, 187 pp.

IEC, 2007: Equipment classification and requirements. Part I, Safety of laser products, 2nd ed., CEI/IEC 60825-1:2007, International Electrotechnical Commission, $199 \mathrm{pp}$.

Jones, J. G., and A. Haynes, 1984: A peakspotter program applied to the analysis of increments in turbulence velocity. Royal Aircraft Establishment Tech. Rep. 84071, 76 pp.

Keohan, C., 2007: Ground-based wind shear detection systems have become vital to safe operations. ICAO J., 62 (2), 16-34.

Lau, S. Y., and C. M. Shun, 2000: Observation of terrain-induced windshear around Hong Kong International Airport under stably stratified conditions. Preprints, Ninth Conf. on Mountain Meteorology, Aspen, CO, Amer. Meteor. Soc., 6.4. [Available online at http://ams.confex.com/ams/htsearch.cgi.]

Lee, O. S. M., and C. M. Shun, 2003: Observation of sea breeze interactions at and near Hong Kong International Airport. Meteor. Appl., 10, 1-9.

Mahapatra, P., 1999: Aviation Weather Surveillance Systems: Advanced Radar and Surface Sensors for Flight Safety and Air Traffic Management. IEE Radar, Sonar, Navigation and Avionics, Series 8, Institution of Electrical Engineers and American Institute of Aeronautics and Astronautics, $453 \mathrm{pp}$.

Michelson, M., W. W. Shrader, and J. G. Wieler, 1990: Terminal Doppler weather radar. Microwave J., 33, 139-148.

Proctor, F. H., D. A. Hinton, and R. L. Bowles, 2000: A windshear hazard index. Preprints, Ninth Conf. on Aviation, Range, and Aerospace Meteorology, Orlando, FL, Amer. Meteor. Soc., 7.7. [Available online at http://ams.confex.com/ams/ htsearch.cgi.]

Shun, C. M., and D. B. Johnson, 1995: Implementation of a terminal Doppler weather radar for the new Hong Kong International Airport at Chek Lap Kok. Preprints, Sixth Conf. on Aviation Weather Systems, Dallas, TX, Amer. Meteor. Soc., 530-534.

, and S. Y. Lau, 2002: Implementation of a Doppler Light Detection and Ranging (LIDAR) system for the Hong Kong International Airport. Preprints, 10th Conf. on Aviation, Range, and Aerospace Meteorology, Portland, OR, Amer. Meteor. Soc., 8.3. [Available online at http://ams.confex.com/ ams/pdfpapers/39018.pdf.]

_ C. M. Cheng, and O. S. M. Lee, 2003a: LIDAR observations of terrain-induced flow and its application in airport windshear monitoring. Proc. Int. Conf. on Alpine Meteorology (ICAM) and Mesoscale Alpine Programme (MAP), Brig, Switzerland, 6.6.

- S. Y. Lau, and O. S. M. Lee, 2003b: Terminal Doppler weather radar observation of atmospheric flow over complex terrain during tropical cyclone passages. J. Appl. Meteor., 42, 1697-1710.

- - - C. M. Cheng, O. S. M. Lee, and H. Y. Chiu, 2004: LIDAR observations of windshear induced by mountain lee waves. Preprints, 11th Conf. on Mountain Meteorology and MAP Meeting 2004, Mount Washington Valley, NH, Amer. Meteor. Soc., 11.2.

Szeto, K. C., and P. W. Chan, 2006: High resolution numerical 
modelling of windshear episodes at the Hong Kong International Airport. Preprints, 12th Conf. on Aviation, Range, and Aerospace Meteorology, Atlanta, GA, Amer. Meteor. Soc., P.5.8. [Available online at http://ams.confex.com/ams/ pdfpapers/100319.pdf.]

Thomson, J. A., and S. M. Hannon, 1995: Wake vortex modeling for airborne and ground-based measurements using a coherent lidar. Air Traffic Control Technologies, R. G. Otto and J. Lenz, Eds., International Society for Optical Engineering (SPIE Proceedings, Vol. 2464), 63-78.
Woodfield, A. A., 1994: Windshear and its effects on aircraft. AGARD Lecture Series on Flight in an Adverse Environment, AGARD LS-197, NATO, 31 pp.

_ , and J. M. Vaughan, 1983: Airspeed and wind shear measurements with an airborne $\mathrm{CO}_{2}$ cw laser. Int. J. Aviation Saf., 1, 207-224.

, and J. F. Woods, 1983: Worldwide experience of windshear during 1981-1982. AGARD Flight Mechanics Panel Conf. on Flight Mechanics and System Design Lessons from Operational Experience, AGARD CP 347, 28 pp. 